\title{
ON REAL INTERPOLATION, FINITE DIFFERENCES, AND ESTIMATES DEPENDING ON A PARAMETER FOR DISCRETIZATIONS OF ELLIPTIC BOUNDARY VALUE PROBLEMS
}

\author{
DAVIDE GUIDETTI AND SERGEI PISKAREV
}

Received 15 April 2003

We give some results concerning the real-interpolation method and finite differences. Next, we apply them to estimate the resolvents of finite-difference discretizations of Dirichlet boundary value problems for elliptic equations in space dimensions one and two in analogs of spaces of continuous and Hölder continuous functions. Such results were employed to study finite-difference discretizations of parabolic equations.

\section{Introduction}

Estimates depending on a parameter for elliptic boundary value problems play a central role not only in evolution equations (see, e.g., [19]) but also in their discretizations, for example, by finite-difference methods (see [3]). In this second case, it is of course of interest to get estimates depending on a parameter for finite-difference analogs of elliptic boundary value problems. In this direction, a significant contribution was given by Alibekov and Sobolevskii in [2]. These authors studied the classical five-point discretization of the second-order problem

$$
\begin{gathered}
\left(\lambda-\sum_{k=1}^{n} a_{k}(x) \frac{\partial^{2}}{\partial x_{k}^{2}}\right) u(x)=f(x), \quad x \in \bar{\Omega}, \\
\gamma_{0} u=0,
\end{gathered}
$$

where $n \in\{1,2\}, \Omega$ equals ]0,1[ or ]0,1[×]0,1[, the functions $a_{k}$ are suitably regular, the operator $\sum_{k=1}^{n} a_{k}(x)\left(\partial^{2} / \partial x_{k}^{2}\right)$ is strongly elliptic, and $\gamma_{0}$ is the trace operator, and they got estimates of the form

$$
\left\|\left(\lambda-A_{h}\right)^{-1}\right\|_{\mathscr{L}\left(\mathscr{C}_{h}\right)} \leq K(1+|\lambda|)^{-1}
$$

Copyright (C) 2003 Hindawi Publishing Corporation Abstract and Applied Analysis 2003:18 (2003) 1005-1035 2000 Mathematics Subject Classification: 65N06, 46B70 URL: http://dx.doi.org/10.1155/S1085337503306359 
Here $\mathscr{C}_{h}$ is a certain class of mesh functions with step $h, A_{h}$ is a discretization of the elliptic operator with Dirichlet boundary conditions in $\mathscr{C}_{h}$, and the positive number $K$ is independent of $h$ and $\lambda$ for $\operatorname{Re} \lambda \geq 0$.

However, the space of continuous functions has an important shortcoming: in a space dimension larger than one, it is not possible to estimate in $C(\bar{\Omega})$ the second-order derivatives of the solution $u$. Therefore, in these spaces, it is more difficult to study problems involving perturbations depending on second-order derivatives, such as parabolic equations which are fully nonlinear or with coefficients depending on time. It is well known that spaces of Hölder continuous functions are much more amenable from this point of view. So we are also interested in estimates depending on $\lambda$ in analogs of spaces of Hölder continuous functions. Estimates depending on a parameter for elliptic boundary value problems were given in $[4,8,9]$ and, under suitable assumptions on the regularity of the coefficients $a_{k}$ and of $\Omega$, are of the form

$$
|\lambda|\|u\|_{C^{\theta}(\bar{\Omega})}+\|u\|_{C^{2+\theta}(\bar{\Omega})} \leq K\left[\|f\|_{C^{\theta}(\bar{\Omega})}+|\lambda|^{\theta / 2}\left\|\gamma_{0} f\right\|_{C(\partial \Omega)}\right]
$$

for $\operatorname{Re} \lambda \geq 0$. In the case of $\Omega=] 0,1[\times] 0,1$, owing to the singularity of the boundary, an estimate of the form (1.3) holds if $f$ vanishes in each vertex (this is a consequence of [9, Proposition 3.1]).

Now, we describe the content of this paper. In Section 2 we put some auxiliary results concerning real-interpolation theory and finite differences. In fact, real-interpolation theory is one of our main tools. We examine in particular one of the possible discretizations of the derivative, the forward difference quotient. This is a bounded operator in all the situations we consider. We establish the uniform equivalence (with respect to the discretization step) of interpolation norms with norms which are discrete versions of norms in Hölder spaces. We conclude showing that a function which has finite difference of a certain order in a certain Banach space and is bounded in another has the intermediate finite differences which are bounded with values in suitable interpolation spaces (Proposition 2.8). This result can be regarded as a discrete version of the theorem by Grisvard (see [6]).

In Section 3, we apply the results of Section 2 to get estimates depending on a parameter for finite-difference discretizations of second-order elliptic boundary value problems in dimension one. First we consider the simplest discretization of the second derivative with Dirichlet boundary condition in a half line; this is necessary for Section 4. Next, we give in Proposition 3.9 a finite-difference analog of estimate (1.3).

In Section 4 we find again, using the foregoing interpolation results and techniques due to Da Prato and Grisvard [5], the essence of the main result in [2] (Theorem 4.3(I)) (in fact, we require a little bit more regular coefficients). Next, we show the uniform equivalence of certain interpolation norms between discrete analogs of the space of continuous functions and the domain of the elliptic operator with Dirichlet boundary conditions with analogs of Hölder norms 
(Theorem 4.3(II)). This is a discrete version of a result due to Labbas [14]. Finally, we indicate a discrete generalization of (1.3) in a square for a problem of the form (1.1).

Some of the results of this paper were also applied to discretizations of parabolic problems in [10, Rothe's scheme] and [11, Crank-Nicolson scheme]. For this purpose, we observe that in the specific case of Hölder continuous functions and their discretizations, estimates of the form (1.3) prevent us from working with infinitesimal generators of operators and so from applying the results of [3]. However, they allow us to get results which are, in some sense, discrete generalizations of certain optimal regularity results which are known for parabolic problems (for this, compare the results of $[10,11]$ with the results of $[8,9]$ ).

We think that the techniques of real interpolation and sums of operators we employ can be useful to treat other problems: for example, discretizations of oblique derivative problems, systems in higher-dimensional parallelepipeds and even in general plane angles (by preliminary change of variables, see [20, Section 4.5]).

We are able to estimate even second-order finite differences of solutions. Concerning the results of this type, we mention again the book [3, Chapter 4] where analogs of estimate depending on a parameter in $\mathbb{R}^{n}$ are given. Estimates in a half space for boundary value problems (not depending on a parameter) were given also by Johnson [13], while Thomée had proved the analogs of interior estimates in [21].

The case of Sobolev-type estimates with $p=2$ in quite general domains (while the estimates not depending on a parameter) was considered in [12, Section 9.2.4]. Results of convergence, being related in some way, were given in [16].

Sobolevskil considered in [18] the heat equation in a rectangular region with discrete analogs of Hölder continuous functions with weights at the boundary in a square and considered in this framework a discretized version of (1.1).

For an introduction to the one-dimensional case in analogs of continuous functions, see also [22].

We conclude this introduction specifying some standard notations we use in the paper.

We indicate with $\mathbb{N}, \mathbb{N}_{0}, \mathbb{Z}, \mathbb{R}, \mathbb{R}^{+}$, and $\mathbb{C}$, respectively, the set of positive and nonnegative integers, the set of integers, the set of real, real positive, and complex numbers. If $\omega \in \mathbb{R}$, we set

$$
[\omega]:=\max \{j \in \mathbb{Z}: j \leq \omega\}, \quad\{\omega\}:=\omega-[\omega] .
$$

If $z$ is a nonvanishing complex number, we indicate with $\operatorname{Arg}(z)$ the element of its argument in the interval ] $-\pi, \pi]$.

An $n$-dimensional multi-index $\alpha$ is, by definition, an element of $\mathbb{N}_{0}^{n}$; if $\alpha=$ $\left(\alpha_{1}, \cdot, \alpha_{n}\right)$, we pose $|\alpha|:=\alpha_{1}+\cdots+\alpha_{n}$.

A linear operator in the Banach space $X$ is an operator of domain $D(A) \subseteq X$ with values in $X$. In this case we indicate with $\rho(A)$ its resolvent set. If $X$ and $Y$ 
are Banach spaces, $\mathscr{L}(X, Y)$ is the Banach space of linear continuous operators from $X$ to $Y$. We omit $Y$ if $Y=X$.

If $A$ and $B$ are elements of $\mathscr{L}(X)$, we set

$$
[A ; B]:=A B-B A
$$

Let $X_{0}$ and $X_{1}$ be Banach spaces with norms, respectively, $\|\cdot\|_{0}$ and $\|\cdot\|_{1}$. We will say that assumption $(h)$ is satisfied if $X_{1} \subseteq X_{0}$ and $\|x\|_{0} \leq\|x\|_{1}$ for every $x \in X_{1}$. Under these conditions, we set, for every $t>0, x \in X_{0}$,

$$
k\left(t, x ; X_{0}, X_{1}\right):=\inf \left\{\|x-y\|_{0}+t\|y\|_{1} \mid y \in X_{1}\right\} .
$$

We write $k(t, x)$ if the spaces $X_{0}$ and $X_{1}$ are clear from the context. If $\left.\theta \in\right] 0,1[$, we define, for $x \in X_{0}$,

$$
\|x\|_{\left(X_{0}, X_{1}\right)_{\theta, \infty}}:=\sup _{t>0} t^{-\theta} k(t, x)
$$

and $\left(X_{0}, X_{1}\right)_{\theta, \infty}:=\left\{x \in X_{0} \mid\|x\|_{\left(X_{0}, X_{1}\right)_{\theta, \infty}}<+\infty\right\}$. In some cases, we will mention even the interpolation space $\left(X_{0}, X_{1}\right)_{\theta, 1}$. For the basic theory concerning these spaces, we refer to [17]. We will write $\left(X_{0}, X_{1}\right)_{\theta}$ instead of $\left(X_{0}, X_{1}\right)_{\theta, \infty}$.

Whenever we consider a space of the form $C^{m}(I, E)$, where $m \in \mathbb{N}_{0}, I$ is an interval in $\mathbb{R}$, and $E$ is a Banach space, we mean that its elements are bounded and uniformly continuous, together with their derivatives of order less than or equal to $m$ and we equip it with its natural norm. We often consider the case that $m \in \mathbb{R}^{+}-\mathbb{N}$, where we assume that the derivative of order $[m]$ is $\{m\}$-Hölder continuous. The same conventions will be used for spaces of the form $C^{s}(\Omega)$, with $s \geq 0$ and $\Omega$ an open subset in $\mathbb{R}^{n}$.

Indicate by $c, c^{\prime}, c_{1}, c_{2}, \ldots$ constants we are not interested to precise, which can be different from time to time. Whenever it is necessary, we will indicate by $c(\theta, \ldots), c^{\prime}(\theta, \ldots), \ldots$ that $c$ (resp. , $\left.c^{\prime}\right)$ depends on $\theta, \ldots$.

Let $X_{0}$ be a Banach space and $A$ a linear operator in $X_{0}$. We say that $A$ satisfies the following condition:

$(\kappa)$ if $] 0,+\infty[\subseteq \rho(A)$ and there exists $M>0$ such that for every $\xi>0$,

$$
\left\|(\xi-A)^{-1}\right\|_{\mathscr{L}\left(X_{0}\right)} \leq M \xi^{-1} .
$$

If $A$ is a closed operator in $X$, we will equip its domain $D(A)$ with the natural norm

$$
\|x\|_{D(A)}:=\max \{\|x\|,\|A x\|\} \quad \forall x \in D(A) .
$$

In this case we will also use the notation $D_{\theta}(A)$ to indicate $(X, D(A))_{\theta}$. 
If $X_{0}, X_{1}$, and $X$ are Banach spaces such that $X_{0}$ and $X_{1}$ are continuously embedded into $X$, if $x \in X_{0} \cap X_{1}$, we set

$$
\|x\|_{X_{0} \cap X_{1}}:=\max \left\{\|x\|_{X_{0}},\|x\|_{X_{1}}\right\} .
$$

\section{The interpolation functor $(\cdot, \cdot)_{\theta, \infty}$ and finite differences}

We start with the following result which is easily proved using the interpolation theory.

Lemma 2.1. Let $X_{0}$ be a Banach space and $A$ a linear operator in $X_{0}$ satisfying condition $(\kappa)$. If $k \in \mathbb{N}$, set $X_{k}:=D\left(A^{k}\right)$ and, if $x \in X_{k},\|x\|_{k}:=\max \left\{\left\|A^{j} x\right\| \mid j \in\right.$ $\{0, \ldots, k\}\}$. Then with this norm, $X_{k}$ is a Banach space. Moreover, for all $k \in \mathbb{N}_{0}$ and $\theta \in] 0,1[$,

$$
\left(X_{k}, X_{k+1}\right)_{\theta}=\left\{x \in X_{k} \mid A^{k} x \in\left(X_{0}, X_{1}\right)_{\theta}\right\}
$$

and there exist $c_{1}, c_{2}$ positive, depending only on $k, \theta$, and $M$ such that for every $x \in\left(X_{k}, X_{k+1}\right)_{\theta}$,

$$
c_{1} \max \left\{\|x\|_{k},\left\|A^{k} x\right\|_{\left(X_{0}, X_{1}\right)_{\theta}}\right\} \leq\|x\|_{\left(X_{k}, X_{k+1}\right)_{\theta}} \leq c_{2} \max \left\{\|x\|_{k},\left\|A^{k} x\right\|_{\left(X_{0}, X_{1}\right)_{\theta}}\right\} .
$$

Now, for $\omega \in \mathbb{R}, \omega>0$, we set

$$
X_{\omega}:=\left\{x \in X_{[\omega]} \mid A^{[\omega]} x \in\left(X_{0}, X_{1}\right)_{\{\omega\}}\right\}
$$

with norm $\|x\|_{\omega}:=\max \left\{\|x\|_{[\omega]},\left\|A^{[\omega]} x\right\|_{\left(X_{0}, X_{1}\right)_{\{\omega\}}}\right\}$.

The following fact will be crucial.

Theorem 2.2. Let $X_{0}$ be a Banach space, $A$ a linear operator in $X_{0}$ satisfying condition $(\kappa)$, and $\theta \in] 0,1[$. Then

(I) $\left(X_{0}, X_{1}\right)_{\theta}=\left\{x \in X_{0} \mid \sup \left\{\xi^{\theta}\left\|A(\xi-A)^{-1} x\right\|_{0} \mid \xi \geq 1\right\}<+\infty\right\}$; moreover, there exist positive constants $c_{1}, c_{2}$ depending only on $M$ such that for every $x \in\left(X_{0}, X_{1}\right)_{\theta}$,

$$
c_{1}\|x\|_{\theta} \leq \max \left\{\|x\|_{0}, \sup \left\{\xi^{\theta}\left\|A(\xi-A)^{-1} x\right\|_{0} \mid \xi \geq 1\right\}\right\} \leq c_{2}\|x\|_{\theta} .
$$

Let $\omega_{0}, \omega_{1}$ be real numbers with $0 \leq \omega_{0}<\omega_{1}$, let $\left.\theta \in\right] 0,1\left[, \omega=(1-\theta) \omega_{0}+\right.$ $\theta \omega_{1}$. Then

(II) if $\omega$ is not an integer,

$$
X_{\omega}=\left(X_{\omega_{0}}, X_{\omega_{1}}\right)_{\theta},
$$

and there exist positive constants $c_{1}, c_{2}$ depending only on $M, \omega_{0}, \omega_{1}, \omega$, and $M$ such that for every $x \in X_{\omega}$,

$$
c_{1}\|x\|_{\omega} \leq\|x\|_{\left(X_{\omega_{0}}, X_{\omega_{1}}\right)_{\theta}} \leq c_{2}\|x\|_{\omega} ;
$$


(III) in any case there exists $c$ positive, depending only on $\omega_{0}, \omega_{1}$, and $\omega$, such that for every $x \in X_{\omega_{1}}$,

$$
\|x\|_{\omega} \leq c\|x\|_{\omega_{0}}^{\left(\omega_{1}-\omega\right) /\left(\omega_{1}-\omega_{0}\right)}\|x\|_{\omega_{1}}^{\left(\omega-\omega_{0}\right) /\left(\omega_{1}-\omega_{0}\right)} .
$$

Proof. See [7, Theorem 3.1, Corollaries 3.1, 3.2].

We now introduce some notations and give some applications of Theorem 2.2 to difference operators. Let $\mathscr{I}$ be a set and $E$ a Banach space with norm $\|\cdot\|$. Giving $U: \mathscr{T} \rightarrow E$ and writing $U_{j}$ instead of $U(j)$ for every $j \in \mathscr{I}$, we set

$$
\begin{gathered}
\|U\|_{B(\Phi, E)}:=\sup _{j \in \mathscr{I}}\left\|U_{j}\right\|, \\
B(\mathscr{I}, E):=\left\{U: \mathscr{I} \longrightarrow E \mid \sup _{j \in \mathscr{I}}\left\|U_{j}\right\|<+\infty\right\} ;
\end{gathered}
$$

it is easily seen that $B(\mathscr{I}, E)$ is a Banach space with the norm $\|\cdot\|_{B(\mathscr{g}, E)}$.

Now assume that $\mathscr{I} \subseteq \mathbb{Z}$; if $U: \mathscr{I} \rightarrow E, j \in \mathscr{I}, j+1 \in \mathscr{I}$, and $h>0$, we set

$$
\partial_{h} U_{j}:=h^{-1}\left(U_{j+1}-U_{j}\right) .
$$

If $m \in \mathbb{N}$, and $j \in \mathscr{I}, j+i \in \mathscr{I}$ for every $i=1, \ldots, m$, we set

$$
\partial_{h}^{m} U_{j}:=h^{-m} \sum_{i=0}^{m}\left(\begin{array}{c}
m \\
i
\end{array}\right)(-1)^{m-i} U_{j+i} .
$$

For $m \in \mathbb{N}$, we put

$$
\mathscr{I}_{m}:=\{j \in \mathscr{I} \mid\{j+1, \ldots, j+m\} \subseteq \mathscr{I}\} .
$$

If $U \in B(\mathscr{I}, E)$, we set

$$
\|U\|_{C_{h}^{m}(\Im, E)}:=\max \left\{\left\|\partial_{h}^{r} U\right\|_{B\left(\Phi_{r}, E\right)} \mid 0 \leq r \leq m\right\},
$$

where, of course, $\mathscr{I}_{0}=\mathscr{I}$ and $\partial_{h}^{0}$ is the identity. Finally, let $\left.\theta \in\right] 0,1[$. We set

$$
[U]_{C_{h}^{\theta}(\mathscr{\Phi}, E)}:=\sup \left\{((k-j) h)^{-\theta}\left\|U_{k}-U_{j}\right\|: j, k \in \Phi, j<k\right\}
$$

and, if $m \in \mathbb{N}_{0}$,

$$
\|U\|_{C_{h}^{m+\theta}(\Im, E)}:=\max \left\{\|U\|_{C_{h}^{m}(\Im, E)},\left[\partial_{h}^{m} U\right]_{C_{h}^{\theta}\left(\Phi_{m}, E\right)}\right\} .
$$

In the same context, we will indicate $B(\Phi, E)$ by $C_{h}^{0}(\Phi, E)$. In each case, if $E$ is omitted, we will assume $E=\mathbb{C}$.

Now consider the space $B(\mathbb{Z}, E)$ and for any $h>0$, the operator $D_{h}$ in $B(\mathbb{Z}, E)$ is defined as $D_{h} U:=\partial_{h} U$. It is clear that $D_{h} \in \mathscr{L}(B(\mathbb{Z}, E))$ and $\|\cdot\|_{D\left(D_{h}\right)}=$ $\|\cdot\|_{C_{h}^{1}(\mathbb{Z}, E)}$ for every $h>0$. We have the following lemma. 
Lemma 2.3. (I) For every $h>0, D_{h}$ satisfies condition $(\kappa)$, with $M=1$;

(II) there exist positive constants $c_{1}, c_{2}$ such that for every $\left.\theta \in\right] 0,1[, h>0$, and $U \in B(\mathbb{Z}, E)$,

$$
c_{1}\|U\|_{C_{h}^{\theta}(\mathbb{Z}, E)} \leq\|U\|_{\left(C(\mathbb{Z}, E), D\left(D_{h}\right)\right)_{\theta}} \leq c_{2}\|U\|_{C_{h}^{\theta}(\mathbb{Z}, E)} .
$$

Proof. (I) By elementary calculations, one has that for every $\xi>0$ and for every $f \in B(\mathbb{Z} ; E)$, the equation

$$
\xi U-D_{h} U=f
$$

has in $B(\mathbb{Z} ; E)$ the unique solution

$$
U_{j}=\sum_{k=j}^{+\infty} G_{h, j-k} f_{k}
$$

where

$$
G_{h, j}= \begin{cases}h(1+h \xi)^{j-1}, & \text { if } j \leq 0 \\ 0, & \text { if } j>0\end{cases}
$$

As $\sum_{j=-\infty}^{0} G_{h, j}=\xi^{-1}$, (I) is proved.

We show (II): let $U \in B(\mathbb{Z} ; E), N:=\|U\|_{\left(B(\mathbb{Z}, E), D\left(D_{h}\right)\right)_{\theta}}$; then, by definition, for every $\epsilon>0$ and for every $t>0$, there exist $V^{t} \in B(\mathbb{Z} ; E)$ such that

$$
\left\|U-V^{t}\right\|_{B(\mathbb{Z} ; E)}+t\left\|V^{t}\right\|_{D\left(D_{h}\right)} \leq(N+\epsilon) t^{\theta} .
$$

Let $j, k \in \mathbb{Z}$ with $j<k$; then,

$$
\begin{aligned}
\left\|U_{k}-U_{j}\right\| & \leq\left\|\left(U_{k}-U_{j}\right)-\left(V_{k}^{t}-V_{j}^{t}\right)\right\|+\left\|V_{k}^{t}-V_{j}^{t}\right\| \\
& \leq 2\left\|U-V^{t}\right\|_{B(\mathbb{Z} ; E)}+(k-j) h\left\|V^{t}\right\|_{D\left(D_{h}\right)} \\
& \leq(N+\epsilon)\left[2 t^{\theta}+(k-j) h t^{\theta-1}\right]
\end{aligned}
$$

so that

$$
[(k-j) h]^{-\theta}\left\|U_{k}-U_{j}\right\| \leq(N+\epsilon)\left\{2 t^{\theta}[(k-j) h]^{-\theta}+[(k-j) h]^{1-\theta} t^{\theta-1}\right\}
$$

for every $t>0$. Choosing $t=(k-j) h$, one gets

$$
[U]_{C_{h}^{\theta}(\mathbb{Z}, E)} \leq 3(N+\epsilon) .
$$


1012 Real interpolation, finite differences, and estimates

To prove the inverse inequality, we use Theorem 2.2(I) and (II). Let $U \in B(\mathbb{Z} ; E)$ and $\xi>0$. Then, after easy computations, for every $k \in \mathbb{Z}$ and $\xi \geq 1$,

$$
\begin{aligned}
& \xi^{\theta}\left\|D_{h}\left(\xi-D_{h}\right)^{-1} U\right\|_{B(\mathbb{Z}, E)} \\
& =\sup _{k \in \mathbb{Z}} \xi^{1+\theta} h\left\|\sum_{r=k}^{\infty}(1+h \xi)^{k-r-1}\left(U_{r}-U_{k}\right)\right\| \\
& \leq N^{\prime}(\xi h)^{1+\theta}(1+h \xi)^{-1} \sum_{r=1}^{+\infty} r^{\theta}(1+h \xi)^{-r} \leq N^{\prime}
\end{aligned}
$$

if $N^{\prime}=[U]_{C_{h}^{\theta}(\mathbb{Z}, E)}$.

As a consequence, we have the following corollary.

Corollary 2.4. Let $\omega_{0}, \omega, \omega_{1} \in \mathbb{R}, 0 \leq \omega_{0}<\omega<\omega_{1}$. Then

(I) if $\omega \notin \mathbb{Z}$, there exist positive constants $c_{1}, c_{2}$ depending on $\omega_{0}$, $\omega$, and $\omega_{1}$ but not on $h$ such that for every $U \in B(\mathbb{Z} ; E)$,

$$
c_{1}\|U\|_{C_{h}^{\omega}(\mathbb{Z}, E)} \leq\|U\|_{\left(C_{h}^{\omega_{0}}(\mathbb{Z}, E), C_{h}^{\omega_{1}}(\mathbb{Z}, E)\right)_{\left(\omega-\omega_{0}\right) /\left(\omega_{1}-\omega_{0}\right)}} \leq c_{2}\|U\|_{C_{h}^{\omega}(\mathbb{Z}, E)} ;
$$

(II) in every case, there exists $c>0$ depending on $\omega_{0}$, $\omega$, and $\omega_{1}$ but not on $h$ such that for every $U \in B(\mathbb{Z} ; E)$,

$$
\|U\|_{C_{h}^{\omega}(\mathbb{Z}, E)} \leq c\|U\|_{C_{h}^{\omega_{0}}(\mathbb{Z}, E)}^{\left(\omega_{1}-\omega\right) /\left(\omega_{1}-\omega_{0}\right)}\|U\|_{C_{h}^{\omega_{1}}(\mathbb{Z}, E)}^{\left(\omega-\omega_{0}\right) /\left(\omega_{1}-\omega_{0}\right)} .
$$

Now consider the space $B\left(\mathbb{N}_{0}, E\right)$ and for any $h>0$ the operator $E_{h}$ in $B\left(\mathbb{N}_{0}, E\right)$ defined as $E_{h} U:=\partial_{h} U$. It is clear that for every $h>0, E_{h} \in \mathscr{L}\left(B\left(\mathbb{N}_{0}, E\right)\right)$ and $\|\cdot\|_{D\left(E_{h}\right)}=\|\cdot\|_{C_{h}^{1}\left(\mathbb{N}_{0}, E\right)}$. With the same methods applied in the case of $D_{h}$, one can show that for every $h>0, E_{h}$ satisfies $(\kappa)$ with $M=1$. Moreover, there exist positive constants $c_{1}, c_{2}$ such that for every $\left.\theta \in\right] 0,1\left[, h>0\right.$ and $U \in B\left(\mathbb{N}_{0}, E\right)$,

$$
c_{1}\|U\|_{C_{h}^{\theta}\left(\mathbb{N}_{0}, E\right)} \leq\|U\|_{\left(B\left(\mathbb{N}_{0}, E\right), D\left(E_{h}\right)\right)_{\theta}} \leq c_{2}\|U\|_{C_{h}^{\theta}\left(\mathbb{N}_{0}, E\right)} .
$$

As a consequence, we have the following proposition.

Proposition 2.5. Let $\omega_{0}, \omega, \omega_{1} \in \mathbb{R}, 0 \leq \omega_{0}<\omega<\omega_{1}$. Then

(I) if $\omega \notin \mathbb{Z}$, there exist positive constants $c_{1}, c_{2}$ depending on $\omega_{0}$, $\omega$, and $\omega_{1}$ but not on $h$ such that for every $U \in B\left(\mathbb{N}_{0} ; E\right)$,

$$
c_{1}\|U\|_{C_{h}^{\omega}\left(\mathbb{N}_{0}, E\right)} \leq\|U\|_{\left(C_{h}^{\omega_{0}}\left(\mathbb{N}_{0}, E\right), C_{h}^{\omega_{1}}\left(\mathbb{N}_{0}, E\right)\right)_{\left(\omega-\omega_{0}\right) /\left(\omega_{1}-\omega_{0}\right)}} \leq c_{2}\|U\|_{C_{h}^{\omega}\left(\mathbb{N}_{0}, E\right)} ;
$$

(II) in every case, there exists $c>0$ depending on $\omega_{0}$, $\omega$, and $\omega_{1}$ but not on $h$ such that for every $U \in B\left(\mathbb{N}_{0} ; E\right)$,

$$
\|U\|_{C_{h}^{\omega}\left(\mathbb{N}_{0}, E\right)} \leq c\|U\|_{C_{h}^{\omega_{0}\left(\mathbb{N}_{0}, E\right)}}^{\left(\omega_{1}-\omega\right) /\left(\omega_{1}-\omega_{0}\right)}\|U\|_{C_{h}^{\left(\omega_{1}\right.}\left(\mathbb{N}_{0}, E\right)}^{\left(\omega-\omega_{0}\right) /\left(\omega_{1}-\omega_{0}\right)} .
$$


Proposition 2.6. Let $L>0, n \in \mathbb{N}, h=L / n, \theta_{0}, \theta, \theta_{1} \in \mathbb{R}$ with $0 \leq \theta_{0}<\theta<\theta_{1}$ and $\theta=(1-\omega) \theta_{0}+\omega \theta_{1}$ for a certain $\left.\omega \in\right] 0,1[$. Then

(I) if $\theta \notin \mathbb{Z}$, there exist positive constants $c_{1}, c_{2}$ depending only on $L, \theta_{0}, \theta$, and $\theta_{1}$ and not on $h$ such that, if $\Phi=\{0, \ldots, n\}$ and $n>\theta_{1}$, for every $f \in$ $B(\Phi, E)$,

$$
c_{1}\|f\|_{C_{h}^{\theta}(\Im, E)} \leq\|f\|_{\left(C_{h}^{\theta_{0}}(\Im, E), C_{h}^{\theta_{1}}(\mathscr{g}, E)\right)_{\omega}} \leq c_{2}\|f\|_{C_{h}^{\theta}(\Im, E)} ;
$$

(II) in any case, there exists $c>0$ depending only on $L, \theta_{0}, \theta$, and $\theta_{1}$ but not on $h$ such that for every $f \in B(\mathscr{\Phi}, E)$,

$$
\|f\|_{C_{h}^{\theta}(\Im, E)} \leq c\|f\|_{C_{h}^{\theta_{0}(\Im, E)}}^{1-\omega}\|f\|_{C_{h}^{\theta_{1}}(\Im, E)}^{\omega} .
$$

Proof. The proof can be obtained by "localizing the estimates" through a partition of unity in $[0, L]$ and applying Corollary 2.4 and Proposition 2.5 (for a similar argument, see the proof of Proposition 3.6).

We conclude the section with a generalization (Proposition 2.8) to finite differences of one of the main results in [6]. It will be used in the fourth section to estimate mixed finite differences of second order. In the proof we will use the following discrete version of Taylor's formula, which can be shown by induction using the fact that for every $l$ and $m$ nonnegative integers,

$$
\sum_{r=0}^{m}\left(\begin{array}{c}
r+l \\
l
\end{array}\right)=\left(\begin{array}{c}
m+l+1 \\
m
\end{array}\right)
$$

Lemma 2.7. Let $\mathscr{I} \subseteq \mathbb{Z}, k \in \mathscr{I}, j, p \in \mathbb{N}$, and $p<j$. Let $U: \mathscr{I} \rightarrow E$ and $h>0$; then, if $k \in \Phi_{j}$,

$$
U_{k+j}=\sum_{l=0}^{p}\left(\begin{array}{l}
j \\
l
\end{array}\right) h^{l} \partial_{h}^{l} U_{k}+h^{p} \sum_{r=0}^{j-p}\left(\begin{array}{c}
j-r-1 \\
p-1
\end{array}\right)\left(\partial_{h}^{p} U_{k+r}-\partial_{h}^{p} U_{k}\right) .
$$

For a similar formula, see [15, Theorem 1.3.4].

Proposition 2.8. Let $I$ be an infinite interval in $\mathbb{R}$ of length $L \in] 0,+\infty], h>0$, $\mathscr{I}:=\{j \in \mathbb{Z} \mid j h \in I\}, \omega>1$, and $\#(\mathscr{S})>[\omega]+1$. Next, let $X_{0}$ and $X_{1}$ be a couple of Banach spaces satisfying assumption $(h)$ and $U \in B\left(\mathscr{I}, X_{1}\right)$. Let $l \in \mathbb{N}, l<\omega$. Then,

$$
\left\|\partial_{h}^{l} U\right\|_{B\left(\Phi_{l},\left(X_{0}, X_{1}\right)_{(\omega-l) / \omega}\right)} \leq c \max \left\{\|U\|_{C_{h}^{\omega}\left(\Im, X_{0}\right)},\|U\|_{B\left(\Im, X_{1}\right)}\right\},
$$

where $c$ is a positive constant depending only on $L, \omega$, and $l$.

Proof. We want to estimate $\left\|\partial_{h}^{l} U_{k}\right\|_{\left(X_{0}, X_{1}\right)_{(\omega-l) / \omega}}$ for a certain $k \in \mathscr{I}_{l}$. Through a translation and a change of orientation, we may assume that $k=0$ and $I \cap$ $\left[0,+\infty\left[\right.\right.$ has length at least equal to $L / 2$. Consider first the case $h(\omega+1)^{2} \geq L / 2$; 
1014 Real interpolation, finite differences, and estimates

then

$$
\begin{aligned}
\left\|\partial_{h}^{l} U_{k}\right\|_{\left(X_{0}, X_{1}\right)_{(\omega-l) / \omega}} & \leq\left\|\partial_{h}^{l} U_{k}\right\|_{1} \leq C(l) h^{-l}\|U\|_{B\left(\Phi, X_{1}\right)} \\
& \leq C(l)\left(\frac{2(\omega+1)^{2}}{L}\right)\|U\|_{B\left(\Im, X_{1}\right)} .
\end{aligned}
$$

Now we assume that

$$
h(\omega+1)^{2}<\frac{L}{2}
$$

so that $\left\{1, \ldots,([\omega]+1)^{2}\right\} \subseteq \mathscr{I}$. Let $x \in \mathbb{N}$ and $x>[\omega]$ such that $[\omega] x \in \mathscr{I}$. By (2.32), for $j=1, \ldots,[\omega]$,

$$
\sum_{l=1}^{[\omega]}\left(\begin{array}{c}
j x \\
l
\end{array}\right) h^{l} \partial_{h}^{l} U_{0}=U_{j x}-U_{0}-h^{[\omega]} \sum_{r=0}^{j x-[\omega]}\left(\begin{array}{c}
j x-r-1 \\
{[\omega]-1}
\end{array}\right)\left(\partial_{h}^{[\omega]} U_{r}-\partial_{h}^{[\omega]} U_{0}\right)
$$

We have

$$
\begin{aligned}
& \operatorname{det}\left(\begin{array}{cccc}
\left(\begin{array}{c}
x \\
1
\end{array}\right) h & \left(\begin{array}{c}
x \\
2
\end{array}\right) h^{2} & \ldots & \left(\begin{array}{c}
x \\
{[\omega]}
\end{array}\right) h^{[\omega]} \\
\left(\begin{array}{c}
2 x \\
1
\end{array}\right) h & \left(\begin{array}{c}
2 x \\
2
\end{array}\right) h^{2} & \cdots & \left(\begin{array}{c}
2 x \\
{[\omega]}
\end{array}\right) h^{[\omega]} \\
\vdots & \vdots & \vdots & \vdots \\
\left(\begin{array}{c}
{[\omega] x} \\
1
\end{array}\right) h & \left(\begin{array}{c}
{[\omega] x} \\
2
\end{array}\right) h^{2} & \ldots & \left(\begin{array}{c}
{[\omega] x} \\
{[\omega]}
\end{array}\right) h^{[\omega]}
\end{array}\right) \\
& =\frac{h^{1+\cdots+[\omega]}}{1 ! 2 ! \cdots[\omega] !} \operatorname{det}\left(\begin{array}{cccc}
x & x^{2} & \cdots & x^{[\omega]} \\
2 x & (2 x)^{2} & \cdots & (2 x)^{[\omega]} \\
\vdots & \vdots & \vdots & \vdots \\
{[\omega] x} & ([\omega] x)^{2} & \cdots & ([\omega] x)^{[\omega]}
\end{array}\right) \\
& =c(\omega)(h x)^{1+\cdots+[\omega]},
\end{aligned}
$$

where $c(\omega)$ is a nonvanishing real number depending only on $\omega$. So from (2.36), we have for $l=1, \ldots,[\omega]$,

$$
\begin{aligned}
& \partial_{h}^{l} U_{0}=\sum_{j=1}^{[\omega]} a_{l j}(h, x)\left(U_{j x}-U_{0}\right) \\
& -h^{[\omega]} \sum_{j=1}^{[\omega]} a_{l j}(h, x)\left[\sum_{r=0}^{j x-[\omega]}\left(\begin{array}{c}
j x-r-1 \\
{[\omega]-1}
\end{array}\right)\left(\partial_{h}^{[\omega]} U_{r}-\partial_{h}^{[\omega]} U_{0}\right)\right],
\end{aligned}
$$


with $\left|a_{l j}(h, x)\right| \leq c(\omega)(h x)^{-l}$ for $1 \leq l$ and $j \leq[\omega]$. This implies that

$$
\begin{aligned}
& \left\|\partial_{h}^{l} U_{0}-\sum_{j=1}^{[\omega]} a_{l j}(h, x)\left(U_{j x}-U_{0}\right)\right\|_{0} \\
& \quad \leq c(\omega) h^{[\omega]}(h x)^{-l}\|U\|_{C_{h}^{\omega}\left(\Im, X_{0}\right)} \sum_{j=1}^{[\omega]} \sum_{r=0}^{j x-[\omega]}\left(\begin{array}{c}
j x-r-1 \\
{[\omega]-1}
\end{array}\right)(r h)^{\{\omega\}} .
\end{aligned}
$$

From (2.31), we have

$$
\begin{aligned}
& \sum_{j=1}^{[\omega]} \sum_{r=0}^{j x-[\omega]}\left(\begin{array}{c}
j x-r-1 \\
{[\omega]-1}
\end{array}\right)(r h)^{\{\omega\}} \\
& \quad \leq h^{\{\omega\}} \sum_{j=1}^{[\omega]}\left(\begin{array}{c}
j x \\
j x-[\omega]
\end{array}\right)(j x-[\omega])^{\{\omega\}} \\
& \quad \leq c(\omega) h^{\{\omega\}} x^{\omega} \\
& \quad \leq c_{1}(\omega) h^{\{\omega\}}(x-[\omega])^{\omega}
\end{aligned}
$$

as $x-[\omega] \geq 1$. We conclude that

$$
\left\|\partial_{h}^{l} U_{0}-\sum_{j=1}^{[\omega]} a_{l j}(h, x)\left(U_{j x}-U_{0}\right)\right\|_{0} \leq c(\omega)(h(x-[\omega]))^{\omega}(h x)^{-l}\|U\|_{C_{h}^{\omega}\left(\Im, X_{0}\right)} .
$$

On the other hand,

$$
\left\|\sum_{j=1}^{[\omega]} a_{l j}(h, x)\left(U_{j x}-U_{0}\right)\right\|_{1} \leq c(\omega)(h x)^{-l}\|U\|_{B\left(\Im, X_{1}\right)} .
$$

Inequalities (2.41) and (2.42) imply that

$$
k\left(t, \partial_{h}^{l} U_{0}\right) \leq c(\omega)\left\{(h(x-[\omega]))^{\omega}(h x)^{-l}\|U\|_{C_{h}^{\omega}\left(\Phi, X_{0}\right)}+t(h x)^{-l}\|U\|_{B\left(\Im, X_{1}\right)}\right\}
$$

for every $t>0$ and for every $x$ with the declared properties.

We now complete the proof under condition (2.35). We want to estimate $t^{-(\omega-l) / \omega} k\left(t, \partial_{h}^{l} U_{0}\right)$ for $t>0$. We distinguish three cases. We start by assuming that

$$
t^{1 / \omega} h^{-1}<1
$$

it follows that

$$
t^{-(\omega-l) / \omega} k\left(t, \partial_{h}^{l} U_{0}\right) \leq t^{l / \omega}\left\|\partial_{h}^{l} U_{0}\right\|_{1} \leq(l+1) t^{l / \omega} h^{-l}\|U\|_{B\left(\Im, X_{1}\right)} \leq(l+1)\|U\|_{B\left(\Im, X_{1}\right)} .
$$


We now assume that

$$
t^{1 / \omega} h^{-1} \geq 1, \quad[\omega]\left([\omega]+\left[t^{1 / \omega} h^{-1}\right]\right) \in \Phi .
$$

We apply (2.43) with $x=[\omega]+\left[t^{1 / \omega} h^{-1}\right]$; we obtain that

$$
t^{-(\omega-l) / \omega} k\left(t, \partial_{h}^{l} U_{0}\right) \leq c(\omega) \max \left\{\|U\|_{C_{h}^{\omega}\left(\Phi, X_{0}\right)},\|U\|_{B\left(\Im, X_{1}\right)}\right\},
$$

observing that $x \geq t^{1 / \omega} h^{-1} \geq\left[t^{1 / \omega} h^{-1}\right]$. Finally, we consider the case

$$
[\omega]\left([\omega]+\left[t^{1 / \omega} h^{-1}\right]\right) \notin \Phi
$$

which implies that $\omega\left(\omega h+t^{1 / \omega}\right)>L / 2$. It follows, using (2.35), that

$$
t^{1 / \omega}>\frac{L}{2 \omega}-\omega h>\frac{L}{2} \frac{2 \omega+1}{\omega(\omega+1)^{2}} .
$$

So

$$
t^{-(\omega-l) / \omega} k\left(t, \partial_{h}^{l} U_{0}\right) \leq\left(\frac{L(2 \omega+1)}{2 \omega(\omega+1)^{2}}\right)^{l-\omega}\left\|\partial_{h}^{l} U_{0}\right\|_{0} \leq\left(\frac{L(2 \omega+1)}{2 \omega(\omega+1)^{2}}\right)^{l-\omega}\|U\|_{C_{h}^{\omega}\left(\mathcal{f}, X_{0}\right)} .
$$

\section{Estimates depending on a parameter for discretizations of elliptic Dirichlet problems in dimension one}

In this section, we want to give estimates depending on a parameter for discretizations of Dirichlet's elliptic boundary value problems in dimension one.

We start by introducing in the space $B(\mathbb{Z} ; E)$, where $E$ is a fixed Banach space with norm $\|\cdot\|$, the operator $F_{h}$ is defined as follows: for $U \in B(\mathbb{Z} ; E), j \in \mathbb{Z}$,

$$
F_{h} U_{j}:=\partial_{h}^{2} U_{j-1}=h^{-2}\left(U_{j+1}-2 U_{j}+U_{j-1}\right) .
$$

The following result is an easy consequence of [2, Chapter 4, Section 1 , Theorem 4].

Lemma 3.1. $\{\lambda \in \mathbb{C}-\{0\}:|\operatorname{Arg} \lambda|<\pi\} \subseteq \rho\left(F_{h}\right)$. Moreover, for every $\phi_{0} \in[0, \pi[$, for every $\omega \geq 0$, there exists $C\left(\phi_{0}, \omega\right)>0$ such that for every $h>0$, for every $\lambda \in \mathbb{C}$, with $|\operatorname{Arg}(\lambda)| \leq \phi_{0}$ and $|\lambda| \geq 1$, and for every $f \in B(\mathbb{Z} ; E)$,

$$
|\lambda|\left\|\left(\lambda-F_{h}\right)^{-1} f\right\|_{C_{h}^{\omega}(\mathbb{Z}, E)}+\left\|\left(\lambda-F_{h}\right)^{-1} f\right\|_{C_{h}^{\omega+2}(\mathbb{Z}, E)} \leq C\left(\phi_{0}, \omega\right)\|f\|_{C_{h}^{\omega}(\mathbb{Z}, E) .}
$$

Now we consider problems in a half line; the first result is the following lemma. 
Lemma 3.2. Consider the problem

$$
\begin{aligned}
\lambda U_{j}-\partial_{h}^{2} U_{j-1} & =0, \quad \text { for } j \geq 1, \\
U_{0} & \in E .
\end{aligned}
$$

Then, for every $\lambda \in \mathbb{C}-\{0\}$, with $|\operatorname{Arg}(\lambda)|<\pi$, and for every $h>0$, for every $U_{0} \in E$, (3.3) has a unique solution $U \in B\left(\mathbb{N}_{0}, E\right)$. Moreover, for every $\omega \in[0,2]$, there exists $C(\omega)>0$ independent of $\lambda, h$, and $U_{0}$ such that

$$
\|U\|_{C_{h}^{\omega}\left(\mathbb{N}_{0}\right)} \leq C(\omega)(1+|\lambda|)^{\omega / 2}\left\|U_{0}\right\| .
$$

Proof. The problem has the unique solution $U_{j}=\alpha^{j} U_{0}\left(j \in \mathbb{N}_{0}\right)$, where $\alpha$ is the only solution with absolute value less than 1 of the algebraic equation

$$
z^{2}-\left(2+\lambda h^{2}\right) z+1=0
$$

Then,

$$
\begin{gathered}
\|U\|_{B\left(\mathbb{N}_{0} ; E\right)}=\left\|U_{0}\right\|, \\
\left\|\partial_{h}^{2} U\right\|_{B\left(\mathbb{N}_{0} ; E\right)} \leq|\lambda|\left\|U_{0}\right\| .
\end{gathered}
$$

So the result follows from (3.6) and Proposition 2.5(II) (which is useful to estimate $\left.\left\|\partial_{h} U\right\|_{B\left(\mathbb{N}_{0} ; E\right)}\right)$.

We now introduce the following notation. Let $f \in B(\mathbb{N} ; E)$; we indicate by $\tilde{f}$ the extension of $f$ to $\mathbb{N}_{0}$ such that $\tilde{f}_{0}=0$.

Consider, for $h>0$, the following operator $B_{h}$ in the space $B(\mathbb{N} ; E)$. For $U \in$ $B(\mathbb{N} ; E)$ and $j \in \mathbb{N}$, we set

$$
B_{h} U_{j}:=\partial_{h}^{2} \tilde{U}_{j-1}
$$

We have the following lemma.

Lemma 3.3. For every $h>0,\{\lambda \in \mathbb{C}-\{0\}|| \operatorname{Arg} \lambda \mid<\pi\} \subseteq \rho\left(B_{h}\right)$. Moreover, for every $\phi_{0} \in\left[0, \pi\left[\right.\right.$, there exists $C\left(\phi_{0}\right)>0$ such that if $|\lambda| \geq 1,|\operatorname{Arg}(\lambda)| \leq \phi_{0}$, for every $h>0$,

$$
\left\|\left(\lambda-B_{h}\right)^{-1}\right\|_{\mathscr{L}(B(\mathbb{N} ; E))} \leq C\left(\phi_{0}\right)|\lambda|^{-1}
$$

Proof. Let $f \in B(\mathbb{N} ; E), \lambda \in \mathbb{C}-\{0\}$, and $|\operatorname{Arg}(\lambda)|<\pi$. Define $g \in B(\mathbb{Z} ; E)$ as follows: for $j \in \mathbb{Z}, g_{j}=f_{j}$ if $j \geq 1$ and $g_{j}=f_{1}$ if $j \leq 0$. Then, $\|g\|_{B(\mathbb{Z} ; E)}$ $=\|f\|_{B(\mathbb{N} ; E)}$. Now let $V:=\left(\lambda-F_{h}\right)^{-1} g$ and let $Z$ be the solution of (3.3) with $U_{0}=-V_{0}$. Then, owing to Lemmas 3.1 and 3.3, equation

$$
\left(\lambda-B_{h}\right) U=f
$$

has a unique solution $U$ and $U_{j}=V_{j}+Z_{j}$ for every $j \in \mathbb{N}$. The final estimate follows from (3.2) and Lemma 3.2. 
1018 Real interpolation, finite differences, and estimates

Given a nonnegative real number $\omega$ and $F \in B(\mathbb{N} ; E)$, we set

$$
\|f\|_{C_{h, 0}^{\omega}(\mathbb{N}, E)}:=\|\tilde{f}\|_{C_{h}^{\omega}\left(\mathbb{N}_{0}, E\right)} .
$$

Our interest in these norms comes from the following lemma.

Lemma 3.4. Let $\theta \in] 0,1\left[\right.$ and $\theta \neq 1 / 2$. Then there exist positive constants $c_{1}, c_{2}$ depending only on $\theta$ such that for every $h>0$ and for every $f \in B(\mathbb{N} ; E)$,

$$
c_{1}\|f\|_{C_{h, 0}^{2 \theta}(\mathbb{N}, E)} \leq\|f\|_{\left(B(\mathbb{N} ; E), D\left(B_{h}\right)\right)_{\theta}} \leq c_{2}\|f\|_{C_{h, 0}^{2 \theta}(\mathbb{N}, E)} .
$$

Proof. The result follows from Proposition 2.5(I) if we are able to show that there exist positive constants $c_{1}, c_{2}$ depending only on $\theta$ such that for every $t>0, U \in$ $B(\mathbb{N}, E)$,

$$
\begin{aligned}
& c_{1} k\left(t, \tilde{U}, B\left(\mathbb{N}_{0}, E\right), C_{h}^{2}\left(\mathbb{N}_{0}, E\right)\right) \leq k\left(t, U, B(\mathbb{N}, E), D\left(B_{h}\right)\right) \\
& \quad \leq c_{2} k\left(t, \tilde{U}, B\left(\mathbb{N}_{0}, E\right), C_{h}^{2}\left(\mathbb{N}_{0}, E\right)\right) .
\end{aligned}
$$

In fact, let $U, g \in B(\mathbb{N}, E)$. Then, for every $t>0$, owing to Proposition 2.5(II),

$$
\begin{aligned}
\| U- & g\left\|_{B(\mathbb{N}, E)}+t\right\| g \|_{D\left(B_{h}\right)} \\
& =\|\tilde{U}-\tilde{g}\|_{B\left(\mathbb{N}_{0}, E\right)}+t \max \left\{\|\tilde{g}\|_{B\left(\mathbb{N}_{0}, E\right)},\left\|\partial_{h}^{2} \tilde{g}\right\|_{B\left(\mathbb{N}_{0}, E\right)}\right\} \\
& \geq c\left(\|\tilde{U}-\tilde{g}\|_{B\left(\mathbb{N}_{0}, E\right)}+t\|\tilde{g}\|_{C_{h}^{2}\left(\mathbb{N}_{0}, E\right)}\right),
\end{aligned}
$$

where $c$ is a positive constant. It follows that

$$
c k\left(t, \tilde{U}, B\left(\mathbb{N}_{0}, E\right), C_{h}^{2}\left(\mathbb{N}_{0}, E\right)\right) \leq k\left(t, U, B(\mathbb{N}, E), D\left(B_{h}\right)\right) .
$$

On the other hand, let $\Phi \in B\left(\mathbb{N}_{0}, E\right)$. Define $\Psi \in B(\mathbb{N}, E), \Psi_{j}=\Phi_{j}-\Phi_{0}$ for every $j \in \mathbb{N}$. Then,

$$
\|U-\Psi\|_{B(\mathbb{N}, E)}+t\|\Psi\|_{D\left(B_{h}\right)} \leq 2\left(\|\tilde{U}-\Phi\|_{B\left(\mathbb{N}_{0}, E\right)}+t\|\Phi\|_{C_{h}^{2}\left(\mathbb{N}_{0}, E\right)}\right) .
$$

It follows that

$$
k\left(t, U, B(\mathbb{N}, E), D\left(B_{h}\right)\right) \leq 2 k\left(t, \tilde{U}, B\left(\mathbb{N}_{0}, E\right), C_{h}^{2}\left(\mathbb{N}_{0}, E\right)\right)
$$

and the result is proved.

The following result is our first discrete version of estimate (1.3).

Proposition 3.5. Let $\left.0<\phi_{0}<\pi, \theta \in\right] 0,2[, \theta \neq 1$, and $r \in[0,2]$. Then there exists $c>0$, depending only on $\phi_{0}, \theta$, and $r$ such that for every $\lambda \in \mathbb{C}$ with $|\lambda| \geq$ 1 and $|\operatorname{Arg}(\lambda)| \leq \phi_{0}$, for every $h>0$, for every $f \in B(\mathbb{N} ; E)$, and for every $F \in$ $B\left(\mathbb{N}_{0}, E\right)$ such that $f=\left.F\right|_{\mathbb{N}}$,

$$
\left\|\left(\lambda-B_{h}\right)^{-1} f\right\|_{C_{h, 0}^{\theta+r}(\mathbb{N}, E)} \leq c|\lambda|^{r / 2-1}\left[\|F\|_{C_{h}^{\theta}\left(\mathbb{N}_{0}, E\right)}+|\lambda|^{\theta / 2}\left\|F_{0}\right\|\right] .
$$


Proof. Owing to Lemmas 3.3 and 3.4, as it is easily seen that

$$
\left\|\left(\lambda-B_{h}\right)^{-1}\right\|_{\mathscr{L}\left(D\left(B_{h}\right)\right)} \leq c\left(\phi_{0}\right)|\lambda|^{-1}
$$

we have that

$$
\left\|\left(\lambda-B_{h}\right)^{-1}\right\|_{\mathscr{L}\left(C_{h, 0}^{\theta}(\mathbb{N}, E)\right)} \leq c\left(\phi_{0}, \theta\right)|\lambda|^{-1}
$$

We set $V:=\left(\lambda-F_{h}\right)^{-1} E f$, where $E$ is the operator defined as follows:

$$
E f_{j}= \begin{cases}f_{j}, & \text { if } j \geq 1, \\ 3 f_{2-j}-2 f_{3-2 j}, & \text { if } j \leq 0 .\end{cases}
$$

It is not difficult to show that for every $\theta \in[0,2]$, there exists $c(\theta)>0$ such that for every $h>0$ and $f \in B(\mathbb{N}, E)$,

$$
\|E f\|_{C_{h}^{\theta}(\mathbb{Z} ; E)} \leq C(\theta)\|f\|_{C_{h}^{\theta}(\mathbb{N} ; E)} .
$$

Let $Z$ be the solution of (3.3) with $U_{0}=-V_{0}$. We put $U:=\left(\lambda-B_{h}\right)^{-1} f$; then $\tilde{U}_{j}=V_{j}+Z_{j}$ for every $j \in \mathbb{N}_{0}$ so that, owing to Lemmas 3.1 and 3.2,

$$
\begin{aligned}
\|U\|_{C_{h, 0}^{\theta}(\mathbb{N}, E)} & \leq\|V\|_{C_{h}^{\theta}(\mathbb{Z}, E)}+\|Z\|_{C_{h}^{\theta}\left(\mathbb{N}_{0}, E\right)} \\
& \leq c\left(\phi_{0}, \theta\right)\left[|\lambda|^{-1}\|f\|_{C_{h}^{\theta}(\mathbb{N}, E)}+|\lambda|^{\theta / 2}\left\|V_{0}\right\|\right] \\
& \leq c\left(\phi_{0}, \theta\right)|\lambda|^{-1}\left[\|f\|_{C_{h}^{\theta}(\mathbb{N}, E)}+|\lambda|^{\theta / 2}\|f\|_{B(\mathbb{N}, E)}\right] .
\end{aligned}
$$

Now observe that if we set $G_{j}=F_{j}-F_{0}$ for $j \in \mathbb{N}$,

$$
\|G\|_{C_{h, 0}^{\theta}(\mathbb{N}, E)} \leq 2\|F\|_{C_{h}^{\theta}\left(\mathbb{N}_{0}, E\right)} .
$$

This implies that if we set $H_{j}:=F_{0}$ for every $j \in \mathbb{N}$, from (3.19) and (3.22),

$$
\begin{aligned}
\|U\|_{C_{h, 0}^{\theta}(\mathbb{N}, E)} & \leq\left\|\left(\lambda-B_{h}\right)^{-1} G\right\|_{C_{h, 0}^{\theta}(\mathbb{N}, E)}+\left\|\left(\lambda-B_{h}\right)^{-1} H\right\|_{C_{h, 0}^{\theta}(\mathbb{N}, E)} \\
& \leq c|\lambda|^{-1}\left[\|F\|_{C_{h}^{\theta}\left(\mathbb{N}_{0}, E\right)}+|\lambda|^{\theta / 2}\left\|F_{0}\right\|\right] .
\end{aligned}
$$

So the result is proved if $r=0$. The case $r=2$ follows from the case $r=0$ using the equation $\partial_{h}^{2} \tilde{U}_{j}=\lambda \tilde{U}_{j+1}+F_{j+1}$ for every $j \in \mathbb{N}_{0}$; the general case follows from Proposition 2.5(II).

Now let $L>0, n \in \mathbb{N}, n \geq 3$, and $h=L / n$. For $j \in \mathscr{I}:=\{1, \ldots, n-1\}$, we have complex numbers $a_{j}, b_{j}, b_{j}^{\prime}$, and $c_{j}$ satisfying the following conditions:

(C1) there exists $v>0$ such that $\operatorname{Re}\left(a_{j}\right) \geq v$ for every $j \in \mathscr{I}$;

(C2) for every $j \in \mathscr{I}, \max \left\{\left|a_{j}\right|,\left|b_{j}\right|,\left|b_{j}^{\prime}\right|,\left|c_{j}\right|\right\} \leq A$ with $A>v$; 
(C3) there exists $\Omega:[0, L] \rightarrow[0,+\infty[$ such that $\Omega(0)=0$ and $\Omega$ is continuous in 0 such that, for $j, k \in \mathscr{I}$ with $j \leq k$,

$$
\left|a_{k}-a_{j}\right| \leq \Omega((k-j) h)
$$

For $\lambda \in \mathbb{C}$, we want to study the following problem:

$$
\begin{gathered}
\lambda U_{j}-a_{j} \partial_{h}^{2} U_{j-1}-b_{j} \partial_{h} U_{j}-b_{j}^{\prime} \partial_{h} U_{j-1}-c_{j} U_{j}=f_{j} \quad \text { for } j=1, \ldots, n-1, \\
U_{0}=U_{n}=0 .
\end{gathered}
$$

To this aim, we set $\bar{\Phi}:=\{0,1, \ldots, n-1, n\}$ and for $U \in B(\Phi, E)$ and $j \in \bar{\Phi}$,

$$
\tilde{U}_{j}= \begin{cases}U_{j}, & \text { if } j \in \mathscr{I}, \\ 0, & \text { if } j \in\{0, n\},\end{cases}
$$

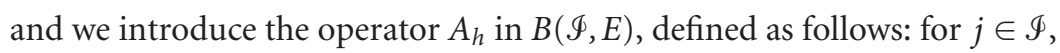

$$
A_{h} U_{j}:=a_{j} \partial_{h}^{2} \tilde{U}_{j-1}+b_{j} \partial_{h} \tilde{U}_{j}+b_{j}^{\prime} \partial_{h} \tilde{U}_{j-1}+c_{j} \tilde{U}_{j}
$$

We have the following proposition.

Proposition 3.6. Assume that assumptions (C1), (C2), and (C3) are satisfied and let $\phi_{0} \in[0, \pi-\arccos (\nu / A)[$. Then, there exist $R>0$ and $M>0$ such that $\left\{\lambda \in \mathbb{C}|| \lambda|\geq R,| \operatorname{Arg}(\lambda) \mid \leq \phi_{0}\right\} \subseteq \rho\left(A_{h}\right)$, and

$$
\left\|\left(\lambda-A_{h}\right)^{-1}\right\|_{\mathscr{L}(B(\mathscr{\Phi}, E))} \leq M|\lambda|^{-1} .
$$

Moreover, for a certain $f \in B(\Phi, E)$, put $U=\left(\lambda-A_{h}\right)^{-1} f$. Then for every $\theta \in$ $[0,2]$, there exists $c>0$ such that

$$
\|\tilde{U}\|_{C_{h}^{\theta}(\bar{g}, E)} \leq c|\lambda|^{\theta / 2-1}\|f\|_{B(\mathscr{\Im}, E)} .
$$

$R$ and $M$ depend only on $L, A, v, \Omega$, and $\phi_{0}$ while $c$ depends only on $L, A, v, \Omega, \phi_{0}$, and $\theta$.

Proof. Fix $\left.\phi_{0} \in\right] \pi / 2, \pi-\arccos (\nu / A)[$. Then, using Lemma 3.3, Proposition 2.5 (II), Lemma 3.1, and a simple perturbation argument, it is easily seen that there exist $\delta \in] 0, L\left[, R_{1}>0\right.$, and $M_{1}>0$ independent of $h$ such that if $U \in B(\mathscr{I}, E)$ and for some $j_{0} \in \mathscr{I} U_{j}=0$ for $\left|\left(j-j_{0}\right) h\right|>\delta, \lambda \in \mathbb{C},|\lambda| \geq R_{1}$, and $|\operatorname{Arg}(\lambda)| \leq \phi_{0}$,

$$
\|\tilde{U}\|_{C_{h}^{\theta}(\bar{\Phi}, E)} \leq M_{1}|\lambda|^{\theta / 2-1}\left\|\left(\lambda-A_{h}\right) U\right\|_{B(\Im, E)} .
$$

Now fix a $C^{\infty}$-partition of unity $\left(\psi_{l}\right)_{1 \leq l \leq P}$ in $[0, L]$ such that for every $l$, the diameter of the support of $\psi^{l}$ is less than or equal to $\delta$. Next, set $\Psi_{j}^{l}:=\psi^{l}(j h)$ for 
every $l$ and for $j \in \bar{\Phi}$. Then, using (3.31), we get for every $U \in B(\mathscr{I}, E)$,

$$
\begin{aligned}
\|\tilde{U}\|_{C_{h}^{\theta}(\bar{\Phi}, E)} & \leq \sum_{l=1}^{P}\left\|\Psi^{l} \tilde{U}\right\|_{C_{h}^{\theta}(\bar{\Phi}, E)} \\
& \leq c_{1}|\lambda|^{\theta / 2-1} \sum_{l=1}^{P}\left\|\left(\lambda-A_{h}\right)\left(\Psi^{l} U\right)\right\|_{B(\Im, E)} \\
& \leq c_{2}|\lambda|^{\theta / 2-1}\left(\|\tilde{U}\|_{C_{h}^{1}(\bar{\Phi}, E)}+\left\|\left(\lambda-A_{h}\right) U\right\|_{B(\Im, E)}\right) .
\end{aligned}
$$

Here $c_{1}$ and $c_{2}$ are constants independent of $h$ and $U$. Considering the case $\theta=1$, one can eliminate $\|\tilde{U}\|_{C_{h}^{1}(\bar{\Phi}, E)}$ in the second term, increasing (if necessary) $R_{1}$.

Equation (3.10) and Lemma 3.4 admit the following natural extensions.

Definition 3.7. Let $\theta \in[0,+\infty[, \theta<n$. If $f \in B(\mathscr{I}, E)$, set

$$
\|f\|_{C_{h, 0}^{\theta}(\Im, E)}:=\|\tilde{f}\|_{C_{h}^{\theta}(\bar{\Im}, E)} .
$$

Proposition 3.8. Assume that assumptions (C1), (C2), and (C3) are satisfied for certain $L, n, v, A$, and $\Omega$. Assume that $n \geq 3$ and let $\theta \in] 0,1[, \theta \neq 1 / 2$. Then, there exist positive constants $c_{1}, c_{2}$ depending only on $L, v, A$, and $\theta$ such that for every $f \in B(\Phi, E)$,

$$
c_{1}\|f\|_{\left(B(\Im, E), D\left(A_{h}\right)\right)_{\theta}} \leq\|f\|_{C_{h, 0}^{2 \theta}(\Phi, E)} \leq c_{2}\|f\|_{\left(B(\Phi, E), D\left(A_{h}\right)\right)_{\theta}} .
$$

Proof. We introduce the operator $B_{h} \in \mathscr{L}(B(\mathscr{I}, E)): B_{h} U_{j}=\partial_{h}^{2} \tilde{U}_{j-1}$ for every $j \in$ $\mathscr{I}$. It is not difficult, using Proposition 2.6, to show the existence of two positive constants $c_{1}, c_{2}$ depending only on $\nu$ and $A$ such that for every $U \in B(\mathscr{I}, E)$,

$$
c_{1}\|U\|_{D\left(B_{h}\right)} \leq\|U\|_{D\left(A_{h}\right)} \leq c_{2}\|U\|_{D\left(B_{h}\right)} .
$$

This means that it suffices to consider the case $A_{h}=B_{h}$ and in this case we can follow the lines of the proof of Proposition 3.5.

Now let $\theta \in] 0,2[$. We introduce the following assumptions: let $L>0, n \in \mathbb{N}$, $n \geq 3$, and $h=L / n$; we set $\mathscr{I}:=\{1, \ldots, n-1\}$ and $\overline{\mathscr{I}}=\{0,1, \ldots, n-1, n\}$. Moreover, $a, b, b^{\prime}$, and $c$ belong to $B(\overline{\mathscr{\Phi}})$. Further, we assume that

(A1) there exists $v>0$ such that $\operatorname{Re}\left(a_{j}\right) \geq v$ for every $j \in \bar{\Phi}$;

(A2) $\max \left\{\|a\|_{C_{h}^{\theta}(\overline{\mathscr{\Im}})},\|b\|_{C_{h}^{\theta}(\overline{\mathcal{S}})},\left\|b^{\prime}\right\|_{C_{h}^{\theta}(\overline{\mathscr{\Im}})},\|b\|_{C_{h}^{\theta}(\overline{\mathscr{\Im}})}\right\} \leq A$ with $A>\nu$.

The following result is our most general discrete version of estimate (1.3) in the one-dimensional case.

Proposition 3.9. Assume that assumptions (A1) and (A2) are satisfied for some $\theta \in] 0,2\left[, \theta \neq 1\right.$. Fix $\phi_{0} \in[0, \pi-\arccos (\nu / A)[$. Then, there exists $R$ positive such that $\left\{\lambda \in \mathbb{C}|| \lambda|\geq R,| \operatorname{Arg}(\lambda) \mid \leq \phi_{0}\right\} \subseteq \rho\left(A_{h}\right)$, where $A_{h}$ is the operator defined in 
1022 Real interpolation, finite differences, and estimates

(3.28). Moreover, for every $r \in[0,2]$, there exists $c>0$ depending only on $L, v, A, r$ such that for every $f \in B(\mathscr{I}, E)$ and for every $F \in B(\bar{\Phi}, E)$ with $\left.F\right|_{\mathscr{I}}=f$,

$$
\left\|\left(\lambda-A_{h}\right)^{-1} f\right\|_{C_{h, 0}^{\theta+r}(\S, E)} \leq c|\lambda|^{r / 2-1}\left[\|F\|_{C_{h}^{\theta}(\bar{\Phi}, E)}+|\lambda|^{\theta / 2} \max \left\{\left\|F_{0}\right\|,\left\|F_{n}\right\|\right\}\right] .
$$

Proof. We start by showing that there exist $\left.\alpha_{1} \in\right] 0,1\left[, R_{1}>0, \phi_{0} \in\right] \pi / 2, \pi[$ such that for every $\lambda \in \mathbb{C}$ with $|\lambda| \geq R_{1}$ and $|\operatorname{Arg}(\lambda)| \leq \phi_{0}$, if $U \in B(\mathscr{S}, E)$ with $U_{j}=0$ for $j h \geq \alpha_{1} L$, for every $r \in[0,2]$,

$$
\|U\|_{C_{h, 0}^{\theta+r}(\Im, E)} \leq c_{1}|\lambda|^{r / 2-1}\left[\|F\|_{C_{h}^{\theta}(\bar{\Phi}, E)}+|\lambda|^{\theta / 2}\left\|F_{0}\right\|\right]
$$

holds for every $F \in B(\overline{\mathscr{I}}, E)$ such that $\left.F\right|_{\mathscr{I}}=\left(\lambda-A_{h}\right) U$, with $c_{1}$ depending only on $A, v, \phi_{0}, \theta, r$. In fact, let $U_{j}=0$ for $j h \geq \alpha L$ for some $\left.\alpha \in\right] 0,1 / 2[$. We put, for $j \in \mathbb{N}_{0}$,

$$
U_{j}^{*}= \begin{cases}\tilde{U}_{j}, & \text { if } j \leq n, \\ 0, & \text { if } j>n .\end{cases}
$$

Assume that $h \leq L / 6$; then, for every $j \in \mathbb{N}$ and $\lambda \in \mathbb{C}$,

$$
\begin{aligned}
\lambda U_{j}^{*} & -a_{0} \partial_{h}^{2} U_{j-1}^{*} \\
& = \begin{cases}\left(a_{j}-a_{0}\right) \partial_{h}^{2} U_{j-1}^{*}+b_{j} \partial_{h} U_{j}^{*}+b_{j}^{\prime} \partial_{h} U_{j-1}^{*}+c_{j} U_{j}^{*}+f_{j}, & \text { if }(j-1) h \leq \alpha L, \\
0, & \text { if }(j-1) h>\alpha L .\end{cases}
\end{aligned}
$$

For $j \in \mathbb{N}$, we now set

$$
G_{j}= \begin{cases}F_{j}+\left(a_{j}-a_{0}\right) \partial_{h}^{2} U_{j-1}^{*}+b_{j} \partial_{h} U_{j}^{*}+b_{j}^{\prime} \partial_{h} U_{j-1}^{*}+c_{j} U_{j}^{*}, & \text { if } j \geq 1,(j-1) h \leq \alpha L, \\ 0, & \text { if }(j-1) h>\alpha L, \\ F_{0}+b_{0} \partial_{h} U_{0}^{*}+b_{0}^{\prime}\left(3 \partial_{h} U_{1}^{*}-2 \partial_{h} U_{2}^{*}\right)+c_{0} U_{0}^{*}, & \text { if } j=0 .\end{cases}
$$

So we have, for $|\lambda| \geq 1,|\operatorname{Arg}(\lambda)| \leq \phi_{0}$, and for every $r \in[0,2]$ (owing to Proposition 3.5),

$$
\|U\|_{C_{h, 0}^{\theta+r}(\Phi, E)}=\left\|U^{*}\right\|_{C_{h}^{\theta+r}\left(\mathbb{N}_{0}, E\right)} \leq c|\lambda|^{r / 2-1}\left[\|G\|_{C_{h}^{\theta}\left(\mathbb{N}_{0}, E\right)}+|\lambda|^{\theta / 2}\left\|G_{0}\right\|\right] .
$$

We have

$$
\|G\|_{C_{h}^{\theta}\left(\mathbb{N}_{0}, E\right)} \leq\|F\|_{C_{h}^{\theta}(\bar{\Phi}, E)}+A(\alpha L)^{\theta^{\prime}}\|\tilde{U}\|_{C_{h}^{2+\theta}(\bar{\Phi}, E)}+c(A)\|\tilde{U}\|_{C_{h}^{2+[\theta]}(\bar{\Phi}, E)},
$$


where $\theta^{\prime}:=\min \{\theta, 1\}$. To get estimate (3.42), consider, for example, the case $\theta \in] 1,2\left[\right.$ and set, for $j \in \mathbb{N}_{0}$,

$$
X_{j}= \begin{cases}\left(a_{j}-a_{0}\right) \partial_{h}^{2} U_{j-1}^{*}, & \text { if } j \geq 1,(j-1) h \leq \alpha L, \\ 0, & \text { otherwise. }\end{cases}
$$

Then, for $j \in \mathbb{N}_{0}$,

$$
\partial_{h} X_{j}= \begin{cases}\partial_{h} a_{j} \partial_{h}^{2} U_{j}^{*}+\left(a_{j}-a_{0}\right) \partial_{h}^{3} U_{j-1}^{*}, & \text { if } 1 \leq j \leq n, \\ \partial_{h} a_{0} \partial_{h}^{2} U_{0}^{*}, & \text { if } j=0, \\ 0, & \text { if }(j-1) h \geq \alpha L\end{cases}
$$

so that

$$
\|X\|_{C_{h}^{\theta}(\bar{\Phi}, E)} \leq A \alpha L\|\tilde{U}\|_{C_{h}^{2+\theta}(\bar{\Phi}, E)}+C(A)\|\tilde{U}\|_{C_{h}^{3}(\bar{\Phi}, E)} .
$$

Next, for example, we put, for $j \in \mathbb{N}_{0}$,

$$
Y_{j}= \begin{cases}b_{j}^{\prime} \partial_{h} U_{j-1}^{*}, & \text { if } 1 \leq j \leq n, \\ b_{0}^{\prime}\left(3 \partial_{h} U_{1}^{*}-2 \partial_{h} U_{2}^{*}\right), & \text { if } j=0 \\ 0, & \text { if } j>n,\end{cases}
$$

and we have

$$
\partial_{h} Y_{j}= \begin{cases}\partial_{h} b_{j}^{\prime} \partial_{h} \tilde{U}_{j}+b_{j}^{\prime} \partial_{h}^{2} \tilde{U}_{j-1}, & \text { if } 1 \leq j \leq n-1, \\ \partial_{h} b_{0}^{\prime} \partial_{h} \tilde{U}_{0}+b_{0}^{\prime}\left(2 \partial_{h}^{2} \tilde{U}_{1}-\partial_{h}^{2} \tilde{U}_{0}\right), & \text { if } j=0, \\ 0, & \text { if }(j-1) h \geq \alpha L,\end{cases}
$$

so that

$$
\|Y\|_{C_{h}^{\theta}(\bar{\Phi}, E)} \leq c(A)\|\tilde{U}\|_{C_{h}^{1+\theta}(\bar{\Im}, E)} .
$$

Next,

$$
\left\|G_{0}\right\| \leq\left\|F_{0}\right\|+c(A)\|\tilde{U}\|_{C_{h}^{1}(\bar{\Phi}, E)} .
$$

So, from (3.42) and (3.49), we get

$$
\begin{aligned}
\|U\|_{C_{h, 0}^{\theta+r}(\Phi, E)} \leq & c(r)|\lambda|^{r / 2-1} \\
\times & {\left[\|F\|_{C_{h}^{\theta}(\bar{\Phi}, E)}+|\lambda|^{\theta / 2}\left\|F_{0}\right\|+A(\alpha L)^{\theta^{\prime}}\|\tilde{U}\|_{C_{h}^{2+\theta}(\bar{\Phi}, E)}\right.} \\
& \left.\quad+c(A)\|\tilde{U}\|_{C_{h}^{2+[\theta]}(\bar{\Phi}, E)}+|\lambda|^{\theta / 2} c(A)\|\tilde{U}\|_{C_{h}^{1}(\bar{\Phi}, E)}\right]
\end{aligned}
$$

and from this estimate, we get (3.37) if $\alpha \leq \alpha_{1}$ and $|\lambda| \geq R_{1}$. Analogously, we can show that there exist $\left.\alpha_{2} \in\right] 0,1\left[\right.$ and $R_{2}>0$ such that for every $\lambda \in \mathbb{C}$ with 
$|\lambda| \geq R_{2}$ and $|\operatorname{Arg}(\lambda)| \leq \phi_{0}$, if $U \in B(\Phi, E)$ with $U_{j}=0$ for $j h \leq\left(1-\alpha_{2}\right) L$,

$$
\|U\|_{C_{h, 0}^{\theta+r}(\Im, E)} \leq c_{2}(r)|\lambda|^{r / 2-1}\left[\|F\|_{C_{h}^{\theta}(\bar{g}, E)}+|\lambda|^{\theta / 2}\left\|F_{n}\right\|\right]
$$

holds for every $F \in B(\overline{\mathscr{T}}, E)$ such that $\left.F\right|_{\mathscr{I}}=\left(\lambda-A_{h}\right) U$. Finally, there exist $\alpha_{3} \in$ ]0,1[ and $R_{3}>0$ such that for every $\lambda \in \mathbb{C}$ with $|\lambda| \geq R_{3}$ and $|\operatorname{Arg}(\lambda)| \leq \phi_{0}$, if $U \in B(\Phi, E)$ with $U_{j}=0$ for $\left|j-j_{0}\right| h \geq \alpha_{3} L$ for some $j_{0}$ such that $\alpha L \leq j_{0} h \leq$ $\left(1-\alpha_{2}\right) L$,

$$
\|U\|_{C_{h, 0}^{\theta+r}(\Im, E)} \leq c_{3}(r)|\lambda|^{r / 2-1}\|F\|_{C_{h}^{\theta}(\bar{\Im}, E)}
$$

holds for every $F \in B(\overline{\mathscr{T}}, E)$ such that $\left.F\right|_{\mathscr{I}}=\left(\lambda-A_{h}\right) U$. Then one can conclude through a partition of unity.

\section{Estimates depending on a parameter for discretizations of the Dirichlet problem for an elliptic equation in a right angle and in a square}

Let $\Omega$ be equal to $] O, L\left[{ }^{2}\right.$ for some $\left.L \in\right] 0,+\infty[$; consider problem (1.1) with $n=2$. We will often write $(x, y)$ instead of $\left(x_{1}, x_{2}\right)$. To study a discretized version of problem (1.1), we start by introducing some notations. Let $\mathscr{I} \subseteq \mathbb{Z}^{2}$ and $V$ : $\mathscr{I} \rightarrow \mathbb{C}$. For $(i, j) \in \mathscr{I}$ and $h>0$, we set

$$
\partial_{x, h} V_{i, j}:=h^{-1}\left(V_{i+1, j}-V_{i, j}\right), \quad \partial_{y, h} V_{i, j}:=h^{-1}\left(V_{i, j+1}-V_{i, j}\right)
$$

if, respectively, $(i+1, j) \in \mathscr{I}$ and $(i, j+1) \in \mathscr{I}$. We can define

$$
\Phi_{(1,0)}:=\{(i, j) \in \mathscr{I} \mid(i+1, j) \in \mathscr{I}\}, \quad \Phi_{(0,1)}:=\{(i, j) \in \mathscr{I} \mid(i, j+1) \in \mathscr{I}\}
$$

and consider the two functions $\partial_{x, h} V$ and $\partial_{y, h} V$ of domains, respectively, $\mathscr{I}_{(1,0)}$ and $\mathscr{I}_{(0,1)}$. If also $(i+1, j+1) \in \mathscr{I}$, even $\partial_{y, h}\left(\partial_{x, h} V\right)_{i, j}$ and $\partial_{x, h}\left(\partial_{y, h} V\right)_{i, j}$ are defined and it is easy to verify that they coincide. More generally, if $\alpha \in \mathbb{N}_{0}^{2}$, we put

$$
\mathscr{I}_{\alpha}:=\left\{(i, j) \in \mathscr{I} \mid(i, j)+\beta \in \mathscr{I} \text { if } \beta \in \mathbb{N}_{0}^{2}, \beta \leq \alpha\right\},
$$

and, for $(i, j) \in \mathscr{I}_{\alpha}$ and $\alpha=\left(\alpha_{1}, \alpha_{2}\right)$,

$$
\partial_{h}^{\alpha} V_{i, j}:=\partial_{y, h}^{\alpha_{2}}\left(\partial_{x, h}^{\alpha_{1}} V\right)_{i, j}
$$

We stress the fact that we can change the order of application of $\partial_{y, h}$ and $\partial_{x, h}$ without changing the result. It is also clear that $\partial_{h}^{\alpha} V$ is a function of domain $\Phi_{\alpha}$.

We now introduce certain norms in $B(\mathscr{I})$, with $\mathscr{I} \subseteq \mathbb{Z}^{2}$ : if $h>0$ and $m \in \mathbb{N}_{0}$, we set, for $V \in B(\mathscr{I})$,

$$
\|V\|_{C_{h}^{m}(\mathscr{I})}:=\max \left\{\left\|\partial_{h}^{\alpha} V\right\|_{B\left(\Im_{\alpha}\right)}\left|\alpha \in \mathbb{N}_{0}^{2},\right| \alpha \mid \leq m\right\} .
$$


Now let $\theta \in] 0,1[$ and $h>0$. If $V \in B(\mathscr{Y})$, we set

$$
\begin{array}{r}
{[V]_{C_{h}^{\theta}(\mathscr{I})}:=\sup \left\{\left\{\left[\left(i_{1}-i_{2}\right)^{2}+\left(j_{1}-j_{2}\right)^{2}\right] h^{2}\right\}^{-\theta / 2}\left|V_{i_{1}, j_{1}}-V_{i_{2}, j_{2}}\right| \mid\left(i_{1}, j_{1}\right) \in \mathscr{I},\right.} \\
\left.\left(i_{2}, j_{2}\right) \in \mathscr{I},\left(i_{1}, j_{1}\right) \neq\left(i_{2}, j_{2}\right)\right\} .
\end{array}
$$

Finally, let $\theta>0$ and $V \in B(\mathscr{I})$. We put

$$
\|V\|_{C_{h}^{\theta(\Im) ~}}:=\max \left\{\|V\|_{C_{h}^{[\theta]}(\mathscr{F})}, \max _{|\alpha|=[\theta]}\left[\partial_{h}^{\alpha} V\right]_{C_{h}^{\{\theta\}}\left(\Phi_{\alpha}\right)}\right\} .
$$

Now let $U \in B\left(\mathbb{N}^{2}\right)$; we indicate by $\tilde{U}$ the element of $B\left(\mathbb{N}_{0}^{2}\right)$ such that

$$
\tilde{U}_{i, j}= \begin{cases}U_{i, j}, & \text { if } \min \{i, j\}>0, \\ 0, & \text { if } \min \{i, j\}=0\end{cases}
$$

We extend (4.8) in the following way: for $n \in \mathbb{N}, n \geq 3$, we set $N_{n}:=\{1, \ldots, n\}$, $N_{0, n}:=N_{n} \cup\{0\}$ and take $h=L / n$. Now let $U \in B\left(N_{n-1}^{2}\right)$; we indicate by $\tilde{U}$ the element of $B\left(N_{0, n}^{2}\right)$ such that

$$
\tilde{U}_{i, j}= \begin{cases}U_{i, j}, & \text { if }(i, j) \in N_{n-1}^{2}, \\ 0, & \text { otherwise. }\end{cases}
$$

We introduce the operators $B_{h}$ and $C_{h}$ in $B\left(N_{n-1}^{2}\right)$ : for $U \in B\left(N_{n-1}^{2}\right),(i, j) \in$ $N_{n-1}^{2}$,

$$
B_{h} U_{i, j}:=b_{i, j} \partial_{x, h}^{2} \tilde{U}_{i-1, j}, \quad C_{h} U_{i, j}:=c_{i, j} \partial_{y, h}^{2} \tilde{U}_{i, j-1},
$$

with $b$ and $c$ real-valued elements of $B\left(N_{0, n}^{2}\right)$. We assume that the following conditions are satisfied:

(a) there exists $v>0$ such that for all $(i, j) \in N_{0, n}^{2}, \min \left\{b_{i, j}, c_{i, j}\right\} \geq v$;

(b) there exists $A>0$ such that for all $i, j \in N_{0, n}$,

$$
\left\|b_{i,}\right\|_{C_{h}^{2}\left(N_{0, n}\right)} \leq A, \quad\left\|c_{\cdot, j}\right\|_{C_{h}^{2}\left(N_{0, n}\right)} \leq A .
$$

Of course, $B_{h}$ and $C_{h}$ belong to $\mathscr{L}\left(B\left(N_{n-1}^{2}\right)\right)$. We introduce the norms $\|\cdot\|_{C_{h, 0}^{\theta}\left(\mathbb{N}_{n-1}^{2}\right)}$ in $B\left(\mathbb{N}_{n-1}^{2}\right)$ : let $\theta \geq 0$ and $U \in B\left(\mathbb{N}_{n-1}^{2}\right)$. We set

$$
\|U\|_{C_{h, 0}^{\theta}\left(\mathbb{N}_{n-1}^{2}\right)}:=\|\tilde{U}\|_{C_{h}^{\theta}\left(\mathbb{N}_{0, n}^{2}\right)} .
$$

We also introduce in $B\left(\mathbb{N}_{n-1}^{2}\right)$, for $h>0$, the operator $A_{h}:=B_{h}+C_{h}$. An easy consequence of Lemma 3.4 and Proposition 3.5 is the following lemma. 
Lemma 4.1. (I) For every $\phi \in[0, \pi[$, there exist $R>0$ and $c(\phi)>0$ such that, for arbitrary $h,\{\lambda \in \mathbb{C}:|\lambda| \geq R,|\operatorname{Arg} \lambda| \leq \phi\} \subseteq \rho\left(B_{h}\right)$ and for these values of $\lambda$,

$$
\left\|\left(\lambda-B_{h}\right)^{-1}\right\|_{\mathscr{L}\left(B\left(\mathbb{N}_{n-1}^{2}\right)\right)} \leq c(\phi)|\lambda|^{-1}
$$

(II) For every $\theta \in] 0,1\left[-\{1 / 2\}\right.$, there exist $c_{1}, c_{2}>0$ depending only on $\theta$ such that for every $U \in B\left(\mathbb{N}_{n-1}^{2}\right)$,

$$
c_{1}\|U\|_{\left(B\left(\mathbb{N}_{n-1}^{2}\right), D\left(B_{h}\right)\right)_{\theta}} \leq \max _{1 \leq j \leq n-1}\|\tilde{U} \cdot, j\|_{C_{h}^{2 \theta}\left(\mathbb{N}_{n-1}\right)} \leq c_{2}\|U\|_{\left(B\left(\mathbb{N}_{n-1}^{2}\right), D\left(B_{h}\right)\right)_{\theta}}
$$

A completely analogous result holds for $C_{h}$, inverting the roles of the variables.

The following theorem will be useful; the first two points follow from [5, Lemma 3.5 and Theorem 3.11], (III) is a particular case of [14, Proposition 2.1], while (IV) is a particular case of [7, Theorem 7.1].

Theorem 4.2. Let $X$ be a Banach space with norm $\|\cdot\|$ and $B$ and $C$ elements of $\mathscr{L}(X)$, satisfying the following assumptions:

(a) there exists $\left.\phi_{0} \in\right] \pi / 2, \pi[$ such that

$$
\left\{\lambda \in \mathbb{C}-\{0\}|| \operatorname{Arg}(\lambda) \mid \leq \phi_{0}\right\} \subseteq \rho(B) \cap \rho(C) ;
$$

(b) for $\lambda \in \mathbb{C}-\{0\}$ with $|\operatorname{Arg}(\lambda)| \leq \phi_{0}$,

$$
\left\|(\lambda-B)^{-1}\right\|_{\mathscr{L}(X)} \leq \frac{M}{|\lambda|}, \quad\left\|(\lambda-C)^{-1}\right\|_{\mathscr{L}(X)} \leq \frac{M}{|\lambda|},
$$

where $M$ is a positive constant;

(c) if $\lambda \in \rho(B)$ and $\mu \in \rho(C)$,

$$
(\lambda-B)^{-1}(\mu-C)^{-1}=(\mu-C)^{-1}(\lambda-B)^{-1} .
$$

Then,

(I) $\left\{\lambda \in \mathbb{C}-\{0\}:|\operatorname{Arg}(\lambda)|<\phi_{0}\right\} \subseteq \rho(B+C)$ and, if $|\operatorname{Arg} \lambda| \leq \phi_{1}<\phi_{0}$,

$$
\left\|(\lambda-B-C)^{-1}\right\|_{\mathscr{L}(X)} \leq C|\lambda|^{-1}
$$

with $C>0$ depending only on $M, \phi_{0}$, and $\phi_{1}$ such that

$$
\left\|(\lambda-B-C)^{-1}\right\|_{\mathscr{L}(X)} \leq C|\lambda|^{-1}
$$


(II) if $\lambda \in \mathbb{C},|\lambda| \geq 1$, and $|\operatorname{Arg} \lambda| \leq \phi_{1}<\phi_{0}$, for every $\left.\theta \in\right] 0,1[$,

$$
\left\|B(\lambda-B-C)^{-1}\right\|_{\mathscr{L}\left(D_{\theta}(B)\right)}+\left\|C(\lambda-B-C)^{-1}\right\|_{\mathscr{L}\left(D_{\theta}(B)\right)} \leq c\left(\phi_{0}, \phi_{1}, M, \theta\right) ;
$$

(III) for every $\theta \in] 0,1\left[\right.$, there exist positive constants $c_{1}, c_{2}$ depending only on $M$ and $\theta$ such that for every $x \in X$,

$$
c_{1}\|x\|_{D_{\theta}(B) \cap D_{\theta}(C)} \leq\|x\|_{D_{\theta}(B+C)} \leq c_{2}\|x\|_{D_{\theta}(B) \cap D_{\theta}(C)} ;
$$

(IV) for every $\theta \in] 0,1\left[\right.$, there exist positive constants $c_{1}, c_{2}$ depending only on $M$ and $\theta$ such that for every $x \in X$,

$$
c_{1}\|x\|_{D_{\theta}(B) \cap D_{\theta}(C)} \leq\|x\|_{(X, D(B) \cap D(C))_{\theta}} \leq c_{2}\|x\|_{D_{\theta}(B) \cap D_{\theta}(C)} .
$$

The following theorem extends [2, Theorem 3] (under slightly more restrictive conditions).

Theorem 4.3. Assume that assumptions (a) and (b) are satisfied. Then

(I) for every $\phi \in[0, \pi[$, there exist $R>0$ and $c(\phi)>0$ such that, for arbitrary $h,\{\lambda \in \mathbb{C}:|\lambda| \geq R,|\operatorname{Arg} \lambda| \leq \phi\} \subseteq \rho\left(A_{h}\right)$ and, for these values of $\lambda$,

$$
\left\|\left(\lambda-A_{h}\right)^{-1}\right\|_{\mathscr{L}\left(B\left(\mathbb{N}_{n-1}^{2}\right)\right)} \leq c(\phi)|\lambda|^{-1}
$$

(II) for every $\theta \in] 0,1\left[-\{1 / 2\}\right.$, there exist positive constants $c_{1}, c_{2}$ depending only on $\theta$ and independent of $h$, such that for every $F \in B\left(\mathbb{N}_{n-1}^{2}\right)$,

$$
c_{1}\|F\|_{\left(B\left(\mathbb{N}_{n-1}^{2}\right), D\left(A_{h}\right)\right)_{\theta}} \leq\|F\|_{C_{h, 0}^{2 \theta}\left(\mathbb{N}_{n-1}^{2}\right)} \leq c_{2}\|F\|_{\left(B\left(\mathbb{N}_{n-1}^{2}\right), D\left(A_{h}\right)\right)_{\theta}} .
$$

Proof. To prove the theorem, we adopt a version of the parametrix method due to Da Prato and Grisvard (see $[5,6]$ ).

Let $\phi \in[0, \pi[$ and $R>0$ as in Lemma 4.1(I). We choose $R$ such that the conclusion of Lemma 4.1(I) holds also for $C_{h}$. Then there exist $\left.\theta_{0} \in\right] 0, \pi[$ and $\delta>$ 0 such that $\Sigma_{\lambda}:=\left\{\mu \in \mathbb{C} \backslash\{0\}:|\operatorname{Arg} \mu|=\theta_{0}\right\} \cup\{\mu \in \mathbb{C}:|\mu| \leq \delta|\lambda|,|\operatorname{Arg} \mu| \leq$ $\left.\theta_{0}\right\} \subseteq \rho\left(\lambda+R-B_{h}\right) \cap \rho\left(C_{h}-R\right)$, for every $\lambda$ such that $|\operatorname{Arg} \lambda| \leq \phi$. We indicate by $\gamma_{\lambda}$ the counterclockwise oriented boundary of $\{\mu \in \mathbb{C} \backslash\{0\}:|\operatorname{Arg} \mu| \leq$ $\left.\phi_{0}\right\} \cup\{\mu \in \mathbb{C}:|\mu| \leq \delta|\lambda|\}$ and we set

$$
S_{\lambda}:=\frac{1}{2 \pi i} \int_{\gamma_{\lambda}}\left(\lambda+R-\mu-B_{h}\right)^{-1}\left(\mu+R-C_{h}\right)^{-1} d \mu .
$$

Applying Cauchy's theorem, we get

$$
\left(\lambda+2 R-A_{h}\right) S_{\lambda}=I-R_{\lambda},
$$


where

$$
R_{\lambda}=\frac{1}{2 \pi i} \int_{\gamma_{\lambda}}\left[C_{h} ;\left(\lambda+R-\mu-B_{h}\right)^{-1}\right]\left(\mu+R-C_{h}\right)^{-1} d \mu
$$

Now let $\mu$ and $\nu$ be complex numbers such that $\max \{|\operatorname{Arg} \mu|,|\operatorname{Arg} \nu|\} \leq \phi^{\prime}<$ $\pi$ and with a suitably large modulus. Then

$$
\left[C_{h} ;\left(\mu-B_{h}\right)^{-1}\right]\left(\nu-C_{h}\right)^{-1}=\left(\mu-B_{h}\right)^{-1}\left[C_{h} ; B_{h}\right]\left(\mu-B_{h}\right)^{-1}\left(\nu-C_{h}\right)^{-1} .
$$

One can verify that for all $U \in B\left(\mathbb{N}_{n-1}^{2}\right)$ and for all $(i, j) \in \mathbb{N}_{n-1}^{2}$,

$$
\begin{aligned}
{\left[C_{h} ; B_{h}\right] U_{i, j}=} & {\left[c_{i-1, j} \partial_{y, h} b_{i, j-1}-b_{i, j-1} \partial_{x, h} c_{i-1, j}\right]\left[\partial_{x, h}^{2} \partial_{y, h} \tilde{U}_{i-1, j-1}-\partial_{x, h}^{2} \partial_{y, h} \tilde{U}_{i-1, j}\right] } \\
& +2 c_{i, j} \partial_{y, h} b_{i, j-1} \partial_{x, h}^{2} \partial_{y, h} \tilde{U}_{i-1, j}+c_{i, j} \partial_{y, h}^{2} b_{i, j-1} \partial_{x, h}^{2} \tilde{U}_{i-1, j-1} \\
& -2 b_{i, j} \partial_{x, h} c_{i-1, j} \partial_{x, h} \partial_{y, h}^{2} \tilde{U}_{i, j-1}-b_{i, j} \partial_{x, h}^{2} c_{i-1, j} \partial_{y, h}^{2} \tilde{U}_{i-1, j-1} .
\end{aligned}
$$

Moreover, if $U=\left(\mu-B_{h}\right)^{-1} V$, for $1 \leq i \leq n-1$ and $0 \leq j \leq n-1$,

$$
\mu \partial_{y, h} \tilde{U}_{i, j}-b_{i, j} \partial_{x, h}^{2} \partial_{y, h} \tilde{U}_{i-1, j}=\partial_{y, h} \tilde{V}_{i, j}+\partial_{y, h} b_{i, j} \partial_{x, h}^{2} \tilde{U}_{i-1, j+1}
$$

and for $1 \leq i \leq n-1$ and $0 \leq j \leq n-2$,

$$
\begin{aligned}
\mu \partial_{y, h}^{2} \tilde{U}_{i, j}-b_{i, j} \partial_{x, h}^{2} \partial_{y, h}^{2} \tilde{U}_{i-1, j}= & \partial_{y, h}^{2} \tilde{V}_{i, j}+\partial_{y, h} b_{i, j} \partial_{x, h}^{2} \partial_{y, h} \tilde{U}_{i-1, j+1} \\
& +\partial_{y, h}^{2} b_{i, j} \partial_{x, h}^{2} \tilde{U}_{i-1, j+2}+\partial_{y, h} b_{i, j} \partial_{x, h}^{2} \partial_{y, h} \tilde{U}_{i-1, j+1} .
\end{aligned}
$$

So, for $|\mu|$ and $|\nu|$ sufficiently large, we obtain, employing also Proposition 3.6,

$$
\left\|\left[C_{h} ;\left(\mu-B_{h}\right)^{-1}\right]\left(\nu-C_{h}\right)^{-1}\right\|_{\mathscr{L}\left(B\left(\mathbb{N}_{n-1}^{2}\right)\right)} \leq C(\phi)|\mu|^{-1}\left[|\mu|^{-1 / 2}+|\nu|^{-1 / 2}\right] .
$$

So we have

$$
\left\|R_{\lambda}\right\|_{\mathscr{L}\left(B\left(\mathbb{N}_{n-1}^{2}\right)\right)} \leq C(\phi) \int_{\gamma_{\lambda}}|\lambda+R-\mu|^{-1}\left(|\lambda+R-\mu|^{-1 / 2}+|\mu+R|^{-1 / 2}\right)|d \mu|
$$

which tends to 0 as $|\lambda|$ tends to $+\infty$, with $|\operatorname{Arg} \lambda| \leq \phi_{1}<\phi_{0}$. We conclude that, for $|\lambda|$ sufficiently large, $\lambda \in \rho\left(A_{h}\right)$ and $\left(\lambda-A_{h}\right)^{-1}=S_{\lambda}\left(1-R_{\lambda}\right)^{-1}$. We have, moreover, for $|\lambda|$ suitably large and $|\operatorname{Arg} \lambda| \leq \phi$,

$$
\left\|\left(\lambda-A_{h}\right)^{-1}\right\|_{\mathscr{L}\left(B\left(\mathbb{N}_{n-1}^{2}\right)\right)} \leq 2|| S_{\lambda} \|_{\mathscr{L}\left(B\left(\mathbb{N}_{n-1}^{2}\right)\right)} \leq C(\phi)|\lambda|^{-1}
$$

with the same method of [5, Lemma 3.5]. With this, (I) is completely proved. 
We show (II). First of all, we observe that, owing to Propositions 2.6, 2.8, and Lemma 4.1, there exist $c_{1}$ and $c_{2}$ positive and independent of $h$ such that for every $F \in B\left(\mathbb{N}_{n-1}^{2}\right)$, if $\left.\theta \in\right] 0,1[\backslash\{1 / 2\}$,

$$
c_{1}\|F\|_{D_{\theta}\left(B_{h}\right) \cap D_{\theta}\left(C_{h}\right)} \leq\|F\|_{C_{h, 0}^{2 \theta}\left(\mathbb{N}_{n-1}^{2}\right)} \leq c_{2}\|F\|_{D_{\theta}\left(B_{h}\right) \cap D_{\theta}\left(C_{h}\right)} .
$$

Now let $\lambda>0$ be sufficiently large so that it belongs to $\rho\left(A_{h}\right)$. Then

$$
\begin{aligned}
\left\|\left(\lambda-A_{h}\right)^{-1}\right\|_{\mathscr{L}\left(B\left(\mathbb{N}_{n-1}^{2}\right), D_{\theta}\left(B_{h}\right)\right)} & \leq 2\left\|S_{\lambda}\right\|_{\mathscr{L}\left(B\left(\mathbb{N}_{n-1}^{2}\right), D_{\theta}\left(B_{h}\right)\right)} \\
& \leq C \int_{\gamma_{\lambda}}(|\lambda+R-\mu|)^{\theta-1}|\mu|^{-1}|d \mu| \\
& \leq C \lambda^{\theta-1} .
\end{aligned}
$$

Reversing the roles of $B_{h}$ and $C_{h}$, one can even show that

$$
\left\|\left(\lambda-A_{h}\right)^{-1}\right\|_{\mathscr{L}\left(B\left(\mathbb{N}_{n-1}^{2}\right), D_{\theta}\left(C_{h}\right)\right)}=O\left(\lambda^{\theta-1}\right)
$$

for $\lambda \rightarrow+\infty$. It follows from [1, Theorem 5.2] that there exists $c>0$ independent of $h$ such that for every $F \in B\left(\mathbb{N}_{n-1}^{2}\right)$,

$$
\|F\|_{D_{\theta}\left(B_{h}\right) \cap D_{\theta}\left(C_{h}\right)} \leq C\|F\|_{\left(B\left(\mathbb{N}_{n-1}^{2}\right), D\left(A_{h}\right)\right)_{\theta, 1}} .
$$

Now indicate by $B_{h}^{0}$ and $C_{h}^{0}$ the operators $B_{h}$ and $C_{h}$ with $b_{i, j}=c_{i, j}=1$ for all $(i, j) \in \mathbb{N}_{0, n}^{2}$. Observe that the norms $\|\cdot\|_{D_{\theta}\left(B_{h}\right) \cap D_{\theta}\left(C_{h}\right)}$ and $\|\cdot\|_{D_{\theta}\left(B_{h}^{0}\right) \cap D_{\theta}\left(C_{h}^{0}\right)}$ are uniformly equivalent. Now as operators $B_{h}^{0}$ and $C_{h}^{0}$ commute, by Theorem 4.2(IV), there exist $c_{1}$ and $c_{2}$ independent of $h$ such that for every $F \in B\left(\mathbb{N}_{n-1}^{2}\right)$,

$$
c_{1}\|F\|_{D_{\theta}\left(B_{h}^{0}\right) \cap D_{\theta}\left(C_{h}^{0}\right)} \leq\|F\|_{\left(B\left(\mathbb{N}_{n-1}^{2}\right), D\left(B_{h}^{0}\right) \cap D\left(C_{h}^{0}\right)\right)_{\theta}} \leq c_{2}\|F\|_{D_{\theta}\left(B_{h}^{0}\right) \cap D_{\theta}\left(C_{h}^{0}\right)} .
$$

But as there, obviously, exists $c>0$ independent of $h$ such that $\|F\|_{D\left(A_{h}\right)} \leq$ $c\|F\|_{D\left(B_{h}^{0}\right) \cap D\left(C_{h}^{0}\right)}$, we obtain that there exist positive constants $c_{1}, c_{2}$ independent of $h$ such that

$$
\|F\|_{D_{\theta}\left(A_{h}\right)} \leq \mathcal{c}_{1}\|F\|_{\left(B\left(\mathbb{N}_{n-1}^{2}\right), D\left(B_{h}^{0}\right) \cap D\left(C_{h}^{0}\right)\right)_{\theta}} \leq \mathcal{c}_{2}\|F\|_{D_{\theta}\left(B_{h}\right) \cap D_{\theta}\left(C_{h}\right)}
$$

for every $F$.

So, by the reiteration property (see $[17,1.2 .3]$ ), there exist $c_{1}$ and $c_{2}$ positive and independent of $h$ such that

$$
\|F\|_{D_{\theta}\left(B_{h}\right) \cap D_{\theta}\left(C_{h}\right)} \leq c_{1}\|F\|_{\left(B\left(\mathbb{N}_{n-1}^{2}\right), D\left(A_{h}\right)\right)_{\theta}} \leq c_{2}\|F\|_{D_{\theta}\left(B_{h}\right) \cap D_{\theta}\left(C_{h}\right)} .
$$

Then the conclusion follows from (4.41) and Proposition 2.8. 
Remark 4.4. Indicate by $\tilde{U}$ the extension of $U \in B\left(\mathbb{N}^{2}\right)$ to $\mathbb{N}_{0}^{2}$, putting $\tilde{U}_{i, j}:=0$ if $i, j=0$, and set $\|U\|_{C_{h, 0}^{\theta}\left(\mathbb{N}^{2}\right)}:=\|\tilde{U}\|_{C_{h}^{\theta}\left(\mathbb{N}^{2}\right)}$. Next, for $U \in B\left(\mathbb{N}^{2}\right)$, put $B_{h} U_{i, j}:=$ $\partial_{x, h}^{2} \tilde{U}_{i-1, j}, C_{h} U_{i, j}:=\partial_{y, h}^{2} \tilde{U}_{i, j-1}$, and $A_{h}:=B_{h}+C_{h}$. Then Theorem 4.3 holds if we replace $B\left(\mathbb{N}_{n-1}^{2}\right)$ by $B\left(\mathbb{N}^{2}\right)$ and $\|\cdot\|_{C_{h, 0}^{2 \theta}\left(\mathbb{N}_{n-1}^{2}\right)}$ by $\|\cdot\|_{C_{h, 0}^{\theta}\left(\mathbb{N}^{2}\right)}$. The proof is the same with some simplifications.

We prove estimates depending on a parameter of Schauder type. In the following, we will use the notations

$$
\partial \mathbb{N}_{0}^{2}:=\left\{(i, j) \in \mathbb{N}_{0}^{2}: i \cdot j=0\right\}
$$

and, if $n \in \mathbb{N}$,

$$
\partial \mathbb{N}_{0, n}^{2}:=\left\{(i, j) \in \mathbb{N}_{0, n}^{2}: \min \{i, j\}=0 \text { or } \max \{i, j\}=n\right\} .
$$

We start with the following lemma.

Lemma 4.5. Let $A_{h}$ be defined as in Remark 4.4. Let $\left.\theta \in\right] 0,1[$. Then there exists $c>0$ such that for every $G \in B\left(\mathbb{N}_{0}^{2}\right)$, with $G_{0,0}=0$ and for every $h>0$, it is possible to construct $Z \in B\left(\mathbb{N}^{2}\right)$ satisfying the following conditions:

(a) $\|Z\|_{C_{h, 0}^{2+\theta}\left(\mathbb{N}^{2}\right)} \leq c\|G\|_{C_{h}^{\theta}\left(\mathbb{N}_{0}^{2}\right)}$;

(b) $\left\|A_{h} Z+F\right\|_{C_{h, 0}^{\theta}\left(\mathbb{N}^{2}\right)} \leq c\|G\|_{C_{h}^{\theta}\left(\mathbb{N}_{0}^{2}\right)}$, where $F:=\left.G\right|_{\mathbb{N}^{2}}$.

Proof. Let $\Omega:=] 0, \infty\left[{ }^{2}\right.$ and $h>0$. We construct $v \in C^{\theta}(\partial \Omega)$ such that $v(i h, j h)=$ $G_{i, j}$ if $(i, j) \in \partial \mathbb{N}_{0}^{2}$. It suffices to put

$$
v(x, y)= \begin{cases}G_{i, 0}+\left(\frac{x}{h}-i\right)\left(G_{i+1,0}-G_{i, 0}\right), & \text { if } i h \leq x<(i+1) h \text { for } i \in \mathbb{N}_{0}, y=0, \\ G_{0, j}+\left(\frac{y}{h}-j\right)\left(G_{0, j+1}-G_{0, j}\right), & \text { if } j h \leq y<(j+1) h \text { for } j \in \mathbb{N}_{0}, x=0 .\end{cases}
$$

It is not difficult to verify that there exist $c_{1}, c_{2}$ positive and independent of $h$ such that

$$
\|v\|_{C^{\theta}(\partial \Omega)} \leq c\left\|G_{\mid \partial \mathbb{N}_{0}^{2}}\right\|_{C_{h}^{\theta}\left(\partial \mathbb{N}_{0}^{2}\right)}
$$

for some $c>0$ independent of $h$. Now fix $\phi \in \mathscr{D}(\mathbb{R})$ such that $\phi$ is even, $\int_{\mathbb{R}} \phi(\xi) d \xi$ $=1$, and $\phi(x)=1$ for $|x| \leq \delta$, for some $\delta>0$, and set, for $(x, y) \in \mathbb{R}^{2}$,

$$
-Q v(x, y):=\phi(x) \frac{x^{2}}{2} \int_{\mathbb{R}} \phi(\xi) \tilde{v}(0, y+\xi x) d \xi+\phi(y) \frac{y^{2}}{2} \int_{\mathbb{R}} \phi(\xi) \tilde{v}(x+\xi y, 0) d \xi,
$$


where $\tilde{v}$ is the extension of $v$ to $\left\{(x, y) \in \mathbb{R}^{2}: x y=0\right\}$ which is odd in $x$ and $y$. Owing to [17, Subsection 0.3], $Q v \in C^{2+\theta}\left(\mathbb{R}^{2}\right), Q v(x, 0)=Q v(0, y)=0$, and $\left(\partial^{2} Q v / \partial y^{2}\right)(x, 0)=-\tilde{g}(x, 0),\left(\partial^{2} Q v / \partial x^{2}\right)(0, y)=-\tilde{g}(0, y)$, for every $x, y \in \mathbb{R}$. Observe also that

$$
\frac{\partial^{2} Q v}{\partial x^{2}}(x, 0)=\frac{\partial^{2} Q v}{\partial y^{2}}(0, y)=0
$$

for every choice of $x$ and $y$ in $\mathbb{R}$.

Now we set, for $(i, j) \in \mathbb{N}^{2}$,

$$
Z_{i, j}:=Q v(i h, j h)
$$

It is not difficult to verify that there exist $c_{1}>0, c_{2}$ independent of $h$ and $v$ such that

$$
\|Z\|_{C_{h, 0}^{2+\theta}\left(\mathbb{N}^{2}\right)} \leq c_{1}\|Q v\|_{C^{2+\theta}(\bar{\Omega})} \leq c_{2}\left\|G_{\mid \partial \mathbb{N}_{0}^{2}}\right\|_{C_{h}^{\theta}\left(\partial \mathbb{N}_{0}^{2}\right)}
$$

It remains to verify (b). We estimate only, for example, (ih) ${ }^{-\theta}\left|A_{h} Z_{i, j}+F_{i, j}\right|$ for $(i, j) \in \mathbb{N}^{2}$. We have

$$
\begin{aligned}
(i h)^{-\theta}\left|A_{h} Z_{i, j}+F_{i, j}\right| \leq & (i h)^{-\theta}\left|\frac{\tilde{Z}_{i+1, j}-2 \tilde{Z}_{i, j}+\tilde{Z}_{i-1, j}}{h^{2}}+F_{i, j}\right| \\
& +(i h)^{-\theta}\left|\frac{\tilde{Z}_{i, j+1}-2 \tilde{Z}_{i, j}+\tilde{Z}_{i, j-1}}{h^{2}}\right| .
\end{aligned}
$$

As $\left(\partial^{2} Q v / \partial y^{2}\right)(0, z)=0$ for every $z \in[0,+\infty[$, the second summand equals

$$
\begin{aligned}
(i h)^{-\theta} & \left|\frac{Q v(i h,(j+1) h)-2 Q v(i h, j h)+Q v(i h,(j-1) h)}{h^{2}}\right| \\
& =(i h)^{-\theta} h^{-2}\left|\int_{j h}^{(j+1) h}\left(\int_{y-h}^{y}\left[\frac{\partial^{2} Q v}{\partial y^{2}}(i h, z)-\frac{\partial^{2} Q v}{\partial y^{2}}(0, z)\right] d z\right) d y\right| \\
& \leq\|Q v\|_{C^{2+\theta}(\bar{\Omega})} \leq c\|G\|_{C_{h}^{\theta}\left(\mathbb{N}_{0}^{2}\right)} .
\end{aligned}
$$

As $\left(\partial^{2} Q v / \partial x^{2}\right)(0, z)=-v(0, z)$ for every $z \in[0,+\infty[$, the first summand equals

$$
\begin{aligned}
(i h)^{-\theta} & \left|h^{-2} \int_{i h}^{(i+1) h}\left(\int_{x-h}^{x}\left[\frac{\partial^{2} Q v}{\partial x^{2}}(z, j h)-\frac{\partial^{2} Q v}{\partial x^{2}}(0, j h)\right] d z\right) d x+\left(G_{i, j}-G_{0, j}\right)\right| \\
& \leq(i h)^{-\theta} h^{-2} \int_{i h}^{(i+1) h}\left(\int_{x-h}^{x} z^{\theta} d z\right) d x\|Q v\|_{C^{2+\theta}(\bar{\Omega})}+\|G\|_{C_{h}^{\theta}\left(\mathbb{N}_{0}^{2}\right)} \\
& \leq c(\theta)\|Q v\|_{C^{2+\theta}(\bar{\Omega})}+\|G\|_{C_{h}^{\theta}\left(\mathbb{N}_{0}^{2}\right)} \leq c\|G\|_{C_{h}^{\theta}\left(\mathbb{N}_{0}^{2}\right)} .
\end{aligned}
$$


1032 Real interpolation, finite differences, and estimates

Lemma 4.6. Let $A_{h}$ have the meaning of Remark 4.4. Let $\left.\left.\lambda \in \mathbb{C}-\right]-\infty, 0\right], \theta \in$ ]0,1[ and $F \in B\left(\mathbb{N}^{2}\right)$. Moreover, $G \in B\left(\mathbb{N}_{0}^{2}\right)$ such that $\left.G\right|_{\mathbb{N}^{2}}=F$ and $G_{0,0}=0$. Then, for every $\phi_{0} \in\left[0, \pi\left[\right.\right.$ and $r \in[0,2]$, there exists $c\left(\phi_{0}, r\right)>0$ such that if $|\lambda| \geq 1,|\operatorname{Arg}(\lambda)| \leq \phi_{0}$, and $h>0$,

$$
\left\|\left(\lambda-A_{h}\right)^{-1} F\right\|_{C_{h, 0}^{\theta+r}\left(\mathbb{N}^{2}\right)} \leq c\left(\phi_{0}, r\right)|\lambda|^{r / 2-1}\left[\|G\|_{C_{h}^{\theta}\left(\mathbb{N}_{0}^{2}\right)}+|\lambda|^{\theta / 2}\|\gamma G\|_{B\left(\partial \mathbb{N}_{0}^{2}\right)}\right],
$$

with $\gamma G:=\left.G\right|_{\partial \mathbb{N}_{0}^{2}}$.

Proof. We start by showing that

$$
\left\|\left(\lambda-A_{h}\right)^{-1} F\right\|_{C_{h, 0}^{\theta+2}\left(\mathbb{N}^{2}\right)} \leq c\|F\|_{C_{h, 0}^{\theta}\left(\mathbb{N}^{2}\right)}
$$

with $c>0$ independent of $\lambda$, with $|\lambda| \geq 1$, and $|\operatorname{Arg} \lambda| \leq \phi_{0}$. In fact, from Proposition 2.8, Lemma 4.1(II), and Theorem 4.2(II), (III), by interpolation, we have

$$
\begin{aligned}
& \left\|\left(\lambda-A_{h}\right)^{-1} F\right\|_{C_{h, 0}^{\theta+2}\left(\mathbb{N}^{2}\right)} \\
& \leq C\left(\left\|\left(\lambda-A_{h}\right)^{-1} F\right\|_{C_{h, 0}^{\theta}\left(\mathbb{N}^{2}\right)}+\left\|B_{h}\left(\lambda-A_{h}\right)^{-1} F\right\|_{C_{h, 0}^{\theta}\left(\mathbb{N}^{2}\right)}\right. \\
& \left.\quad+\left\|C_{h}\left(\lambda-A_{h}\right)^{-1} F\right\|_{C_{h, 0}^{\theta}\left(\mathbb{N}^{2}\right)}\right) \\
& \leq \mathcal{c}\|F\|_{C_{h, 0}^{\theta}\left(\mathbb{N}^{2}\right)}
\end{aligned}
$$

From Remark 4.4, we also have

$$
\left\|\left(\lambda-A_{h}\right)^{-1} F\right\|_{C_{h, 0}^{\theta}\left(\mathbb{N}^{2}\right)} \leq c|\lambda|^{-1}\|F\|_{C_{h, 0}^{\theta}\left(\mathbb{N}^{2}\right)}
$$

So, interpolating between (4.54) and (4.56), we obtain

$$
\left\|\left(\lambda-A_{h}\right)^{-1} F\right\|_{C_{h, 0}^{r+\theta}\left(\mathbb{N}^{2}\right)} \leq c(r)|\lambda|^{r / 2-1}\|F\|_{C_{h, 0}^{\theta}\left(\mathbb{N}^{2}\right)}
$$

for every $r \in[0,2]$. Now we prove that if $|\lambda|=1,|\operatorname{Arg}(\lambda)| \leq \phi_{0}<\pi$, and $U:=$ $\left(\lambda-A_{h}\right)^{-1} F$, then

$$
\|U\|_{C_{h, 0}^{\theta+2}\left(\mathbb{N}^{2}\right)} \leq c\|G\|_{C_{h}^{\theta}\left(\mathbb{N}_{0}^{2}\right)}
$$

with $c>0$ depending only on $\phi_{0}$. In fact, let $Z$ be the element of $B\left(\mathbb{N}^{2}\right)$ constructed in Lemma 4.5. As

$$
\lambda(U-Z)-A_{h}(U-Z)=F+A_{h} Z-\lambda Z
$$


it follows from Lemma 4.5 and (4.54) that

$$
\begin{aligned}
\|U\|_{C_{h, 0}^{\theta+2}\left(\mathbb{N}^{2}\right)} & \leq\|U-Z\|_{C_{h, 0}^{\theta+2}\left(\mathbb{N}^{2}\right)}+\|Z\|_{C_{h, 0}^{\theta+2}\left(\mathbb{N}^{2}\right)} \\
& \leq c_{1}\left\|F+A_{h} Z-\lambda Z\right\|_{C_{h, 0}^{\theta}\left(\mathbb{N}^{2}\right)}+\|Z\|_{C_{h, 0}^{\theta+2}\left(\mathbb{N}^{2}\right)} \\
& \leq c_{2}\left[\|G\|_{C_{h}^{\theta}\left(\mathbb{N}_{0}^{2}\right)}+\|Z\|_{C_{h, 0}^{\theta+2}\left(\mathbb{N}^{2}\right)}\right] \leq c_{3}\|G\|_{C_{h}^{\theta}\left(\mathbb{N}_{0}^{2}\right)} .
\end{aligned}
$$

Now let $\lambda=\rho^{2} e^{i \phi}$ with $\rho \geq 1$ and $|\phi| \leq \phi_{0}$; then, if we continue to indicate by $U$ the element $\left(\lambda-A_{h}\right)^{-1} F$, we have

$$
e^{i \phi} U-A_{h \rho} U=\rho^{-2} F
$$

so that

$$
\|U\|_{C_{h \rho, 0}^{\theta+r}\left(\mathbb{N}^{2}\right)} \leq c(r) \rho^{-2}\|G\|_{C_{h \rho}^{\theta}\left(\mathbb{N}_{0}^{2}\right)}
$$

for every $r \in[0,2]$. A simple consequence of $(4.30)$ is

$$
\left\|\left(\lambda-A_{h}\right)^{-1} F\right\|_{C_{h, 0}^{\theta+r}\left(\mathbb{N}^{2}\right)} \leq c\left(\phi_{0}, r\right)|\lambda|^{r / 2-1}\left[\|G\|_{C_{h}^{\theta}\left(\mathbb{N}_{0}^{2}\right)}+|\lambda|^{\theta / 2}\|G\|_{B\left(N_{0}^{2}\right)}\right] .
$$

Now we set, for $(i, j) \in \mathbb{N}_{0}^{2}$,

$$
H_{i, j}:=G_{i, 0}+G_{0, j}
$$

It is not difficult to verify that

$$
\left.\left\|F-\left.H\right|_{\mathbb{N}^{2}}\right\|\right|_{C_{h, 0}^{\theta}\left(\mathbb{N}^{2}\right)}+\|H\|_{C_{h}^{\theta}\left(\mathbb{N}_{0}^{2}\right)} \leq c\|G\|_{C_{h}^{\theta}\left(\mathbb{N}_{0}^{2}\right)} .
$$

It follows from (4.57) and (4.63) that, for $r \in[0,2]$,

$$
\begin{aligned}
\|(\lambda- & \left.A_{h}\right)^{-1} F \|_{C_{h, 0}^{\theta+r}\left(\mathbb{N}^{2}\right)} \\
& \leq\left\|\left(\lambda-A_{h}\right)^{-1}\left(F-\left.H\right|_{\mathbb{N}^{2}}\right)\right\|_{C_{h, 0}^{\theta+r}\left(\mathbb{N}^{2}\right)}+\left\|\left.\left(\lambda-A_{h}\right)^{-1} H\right|_{\mathbb{N}^{2}}\right\|_{C_{h, 0}^{\theta+r}\left(\mathbb{N}^{2}\right)} \\
& \leq c\left(\phi_{0}, r\right)|\lambda|^{r / 2-1}\left[\left.\left\|F-\left.H\right|_{\mathbb{N}^{2}}\right\|\right|_{C_{h, 0}^{\theta}\left(\mathbb{N}^{2}\right)}+\|H\|_{C_{h}^{\theta}\left(\mathbb{N}_{0}^{2}\right)}+|\lambda|^{\theta / 2}\|H\|_{B\left(\mathbb{N}_{0}^{2}\right)}\right] \\
& \leq c\left(\phi_{0}, r\right)|\lambda|^{r / 2-1}\left[\|G\|_{C_{h}^{\theta}\left(\mathbb{N}_{0}^{2}\right)}+|\lambda|^{\theta / 2}\|\gamma G\|_{B\left(\partial N_{0}^{2}\right)}\right] .
\end{aligned}
$$

Theorem 4.7. Assume that conditions (a) and (b) are satisfied and let $\theta \in] 0,1[$. Assume, moreover, that for all $i, j \in N_{0, n}$,

$$
\|b \cdot j\|_{C_{h}^{\theta}\left(N_{0, n}\right)} \leq A, \quad\left\|c_{i \cdot}\right\|_{C_{h}^{2}\left(N_{0, n}\right)} \leq A .
$$


Let $\lambda \in \mathbb{C}-]-\infty, 0]$ and $F \in B\left(N_{n-1}^{2}\right)$. Let $G \in B\left(N_{0, n}^{2}\right)$ such that $G_{\mid N_{n-1}^{2}}=F$ and $G_{0,0}=G_{n, 0}=G_{n, n}=G_{0, n}=0$. Then, for every $\phi_{0} \in[0, \pi[$ and $r \in[0,2]$, there exists $c\left(\phi_{0}, r\right)>0$ independent of $F$ and $G$ such that if $|\operatorname{Arg}(\lambda)| \leq \phi_{0},|\lambda| \geq 1$, and $h>0$,

$$
\begin{aligned}
\left\|\left(\lambda-A_{h}\right)^{-1} F\right\|_{C_{h, 0}^{\theta+r}\left(N_{n-1}^{2}\right)} \leq & c\left(\phi_{0}, r, A\right)(1+|\lambda|)^{r / 2-1} \\
& \times\left[\|G\|_{C_{h}^{\theta}\left(N_{0, n}^{2}\right)}+(1+|\lambda|)^{\theta / 2}\|\gamma G\|_{B\left(\partial N_{0, n}^{2}\right)}\right] .
\end{aligned}
$$

Proof. The proof can be obtained by the same method of the proof of Proposition 3.9, using Lemma 4.6.

\section{References}

[1] J. O.-O. Adeyeye, Characterisation of real interpolation spaces between the domain of the Laplace operator and $L_{p}(\Omega) ; \Omega$ polygonal and applications, J. Math. Pures Appl. (9) 67 (1988), no. 3, 263-290.

[2] K. A. Alibekov and P. E. Sobolevskiŭ, Stability and convergence of high-order difference schemes for parabolic partial differential equations, Ukrainian Math. J. 32 (1980), no. 3, 189-196.

[3] A. Ashyralyev and P. E. Sobolevskiü, Well-Posedness of Parabolic Difference Equations, Operator Theory: Advances and Applications, vol. 69, Birkhäuser Verlag, Basel, 1994.

[4] P. Bolley, J. Camus, and P. The Lại, Estimation de la résolvante du problème de Dirichlet dans les espaces de Hölder, C. R. Acad. Sci. Paris Sér. I Math. 305 (1987), no. 6, 253-256.

[5] G. Da Prato and P. Grisvard, Sommes d'opérateurs linéaires et équations différentielles opérationnelles, J. Math. Pures Appl. (9) 54 (1975), no. 3, 305-387.

[6] P. Grisvard, Identités entre espaces de traces, Math. Scand. 13 (1963), 70-74.

[7] Commutativité de deux foncteurs d'interpolation et applications, J. Math. Pures Appl. (9) 45 (1966), 143-206.

[8] D. Guidetti, Optimal regularity for mixed parabolic problems in spaces of functions which are Hölder continuous with respect to space variables, Ann. Scuola Norm. Sup. Pisa Cl. Sci. (4) 26 (1998), no. 4, 763-790.

[9] The mixed Cauchy-Dirichlet problem for the heat equation in a plane angle in spaces of Hölder-continuous functions, Adv. Differential Equations 6 (2001), no. 8, 897-930.

[10] D. Guidetti and S. Piskarev, Stability of Rothe's scheme and maximal regularity for parabolic equations in $C^{\theta}(\bar{\Omega})$ spaces, Progress in Partial Differential Equations, Vol. 1 (Pont-à-Mousson, 1997) (H. Amann, C. Bandle, M. Chipot, F. Conrad, and I. Shafrir, eds.), Pitman Res. Notes Math. Ser., vol. 383, Longman, Harlow, 1998, pp. 167-180.

[11] Stability of the Crank-Nicolson scheme and maximal regularity for parabolic equations in $C^{\theta}(\bar{\Omega})$ spaces, Numer. Funct. Anal. Optim. 20 (1999), no. 3-4, 251277.

[12] W. Hackbusch, Elliptic Differential Equations. Theory and Numerical Treatment, Springer Series in Computational Mathematics, vol. 18, Springer-Verlag, Berlin, 1992. 
[13] C. G. L. Johnson, Estimates near plane portions of the boundary for discrete elliptic boundary problems, Math. Comp. 28 (1974), no. 128, 909-935.

[14] R. Labbas, Some results on the sum of linear operators with nondense domains, Ann. Mat. Pura Appl. (4) 154 (1989), 91-97.

[15] V. Lakshmikantham and D. Trigiante, Theory of Difference Equations. Numerical Methods and Applications, Mathematics in Science and Engineering, vol. 181, Academic Press, Massachusetts, 1988.

[16] R. D. Lazarov, V. L. Makarov, and A. A. Samarskii, Application of exact difference schemes to the construction and study of difference schemes for generalized solutions, Math. USSR-Sb. 45 (1983), no. 4, 461-471.

[17] A. Lunardi, Analytic Semigroups and Optimal Regularity in Parabolic Problems, Progress in Nonlinear Differential Equations and Their Applications, vol. 16, Birkhäuser Verlag, Basel, 1995.

[18] P. E. Sobolevskiŭ, On coercive solvability of difference equations, Soviet Math. Dokl. 12 (1971), no. 6, 1802-1805.

[19] H. Tanabe, Equations of Evolution, Monographs and Studies in Mathematics, vol. 6, Pitman, Massachusetts, 1979.

[20] J. W. Thomas, Numerical Partial Differential Equations: Finite Difference Methods, Texts in Applied Mathematics, vol. 22, Springer-Verlag, New York, 1995.

[21] V. Thomée, Discrete interior Schauder estimates for elliptic difference operators., SIAM J. Numer. Anal. 5 (1968), 626-645.

[22] G. Vainikko, Funktionalanalysis der Diskretisierungsmethoden, Teubner-Texte zur Mathematik, B. G. Teubner Verlag, Leipzig, 1976.

Davide Guidetti: Dipartimento di Matematica, Universitá di Bologna, Piazza di Porta S. Donato 5, 40126 Bologna, Italy

E-mail address: guidetti@dm.unibo.it

Sergei Piskarev: Science Research Computer Center, Moscow State University, Moscow 119899, Russia

E-mail address: serguei@piskarev.srcc.msu.ru 


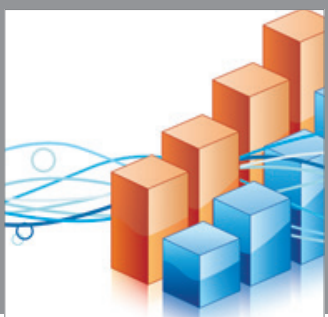

Advances in

Operations Research

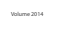

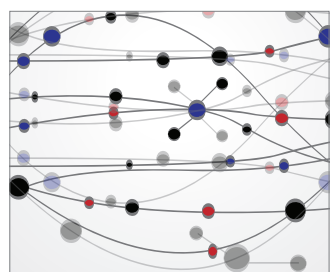

\section{The Scientific} World Journal
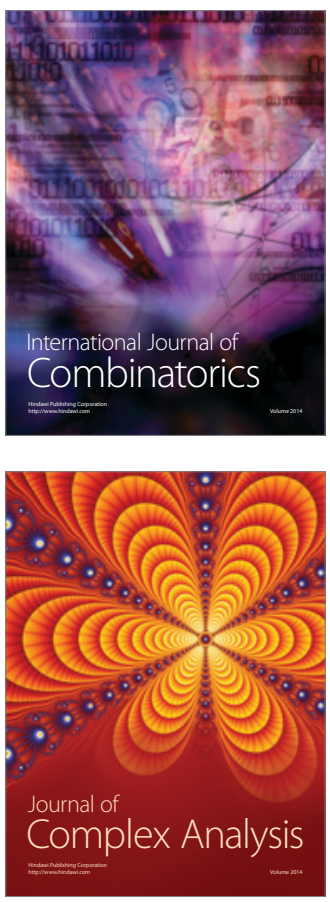

International Journal of

Mathematics and

Mathematical

Sciences
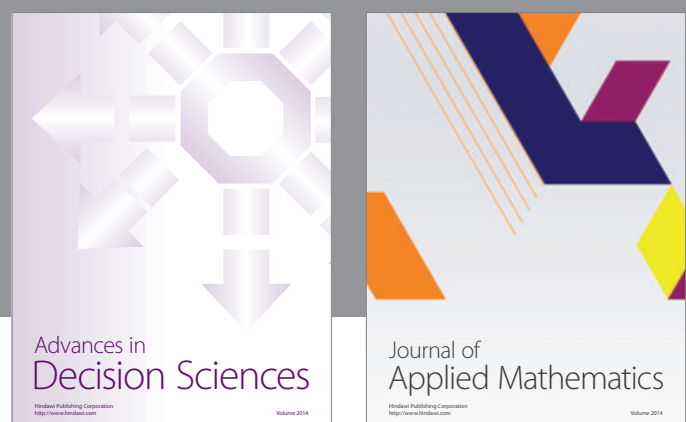

Journal of

Applied Mathematics
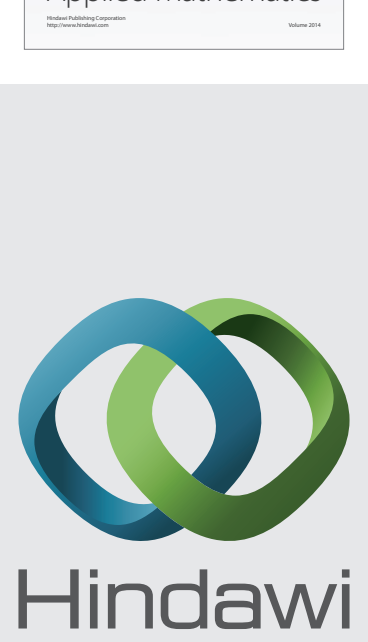

Submit your manuscripts at http://www.hindawi.com
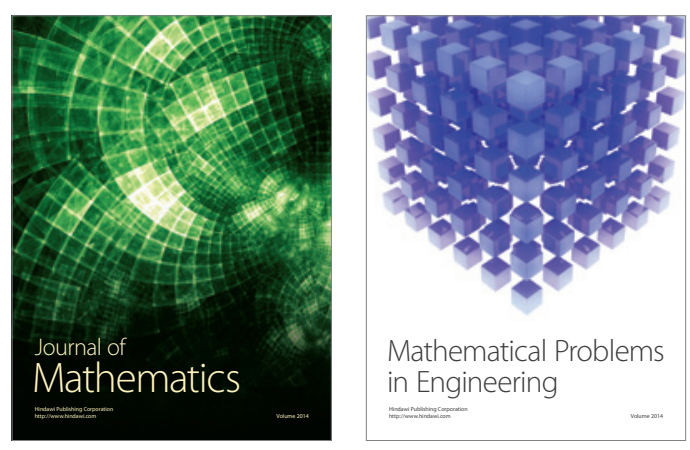

Mathematical Problems in Engineering
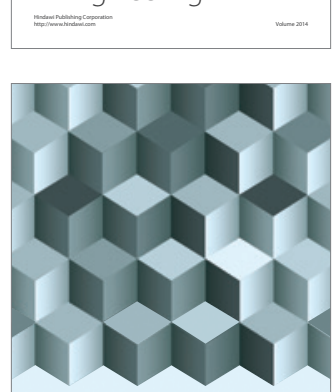

Journal of

Function Spaces
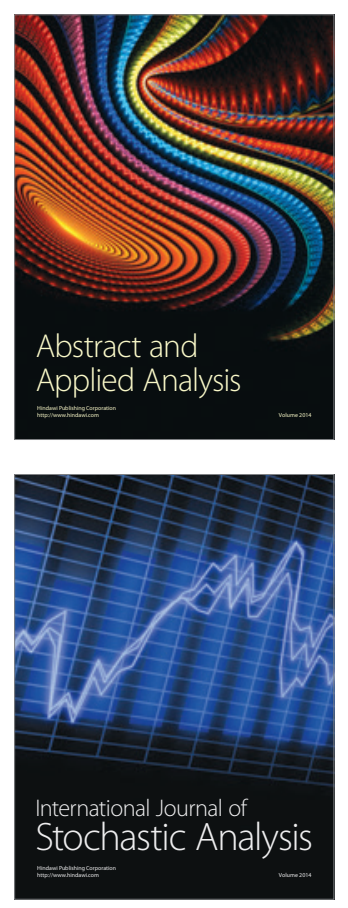

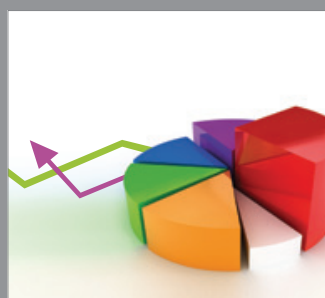

ournal of

Probability and Statistics

Promensencen
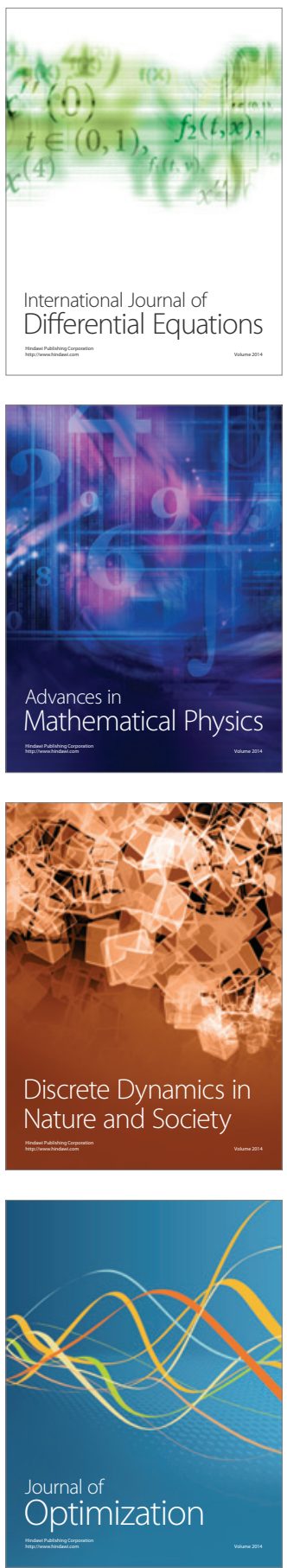